TRANSACTIONS OF THE

AMERICAN MATHEMATICAL SOCIETY

Volume 359, Number 9, September 2007, Pages 4209-4235

S 0002-9947(07)04229-8

Article electronically published on April 11, 2007

\title{
SINGULAR COSPHERE BUNDLE REDUCTION
}

\author{
OANA M. DRĂGULETE, TUDOR S. RATIU, AND MIGUEL RODRÍGUEZ-OLMOS
}

\begin{abstract}
This paper studies singular contact reduction for cosphere bundles at the zero value of the momentum map. A stratification of the singular quotient, finer than the contact one and better adapted to the bundle structure of the problem, is obtained. The strata of this new stratification are a collection of cosphere bundles and coisotropic or Legendrian submanifolds of their corresponding contact components.
\end{abstract}

\section{INTRODUCTION}

The main goal of this paper is to carry out the singular reduction of cosphere bundles at the zero value of the contact momentum map. This presents interest because cosphere bundles carry considerably more structure than a general contact manifold and have the same privileged position in contact geometry that cotangent bundles have in symplectic geometry. They have been intensively used in topological problems dealing with the classification of immersions and embeddings. Associating to each immersion (embedding) of a smooth manifold a Legendrian submanifold in its cosphere bundle one can use Legendrian contact homology to construct topological invariants. A beautiful introduction to the applications of these contact constructions is 7 .

Contact reduction appears for the first time in the work of Guillemin and Sternberg [10] in the context of reducing symplectic cones. Albert [1] and, several years later, Geiges 8 and Loose 13 independently defined and studied contact reduction at the zero value of the contact momentum map for free proper contact actions of Lie groups. Reduction at a general value of the momentum map was studied by both Albert [1] and Willett [20] who proposed two different versions of dealing with it. It turns out that Willett's method is the one that naturally parallels the symplectic reduction theory, even in the singular case as shown by Lerman and Willett [12. They prove that the resulting contact quotient depends only on the contact structure, that it is independent of any contact form defining the contact foliation, and that it is a stratified space, more precisely, a cone space. For an extension

Received by the editors June 30, 2005.

2000 Mathematics Subject Classification. Primary 53D10, 53D20.

Key words and phrases. Contact manifold, cotangent, cosphere bundle, momentum map, singular reduction.

The first and the second authors thank the Swiss National Science Foundation for partial support.

(C)2007 American Mathematical Society

Reverts to public domain 28 years from publication 
of Willett's method, where the contact space can be defined at any value of the momentum map, see [21].

The case of cosphere bundle reduction for proper free lifted Lie group actions was studied in [5] with a view of comparing the theory to that for cotangent bundle reduction. It turns out that in regular contact reduction of cosphere bundles there are no analogues of magnetic terms. In parallel, in 17 the authors have developed the theory of singular cotangent bundle reduction at the zero value of the momentum map and have found a finer stratification than that given by the general theory due to the additional structure of the cotangent bundle and the fact that the Lie group action is a cotangent lifted action. A similar phenomenon occurs in contact reduction of cosphere bundles. Applying the general theory of singular contact reduction due to Lerman and Willett [12] yields contact stratified spaces that, however, lose all information of the internal structure of the cosphere bundle. Based on the cotangent bundle reduction theorems, both in the regular and singular cases, as well as regular cosphere bundle reduction, one expects additional bundle-like structure for the contact strata. The cosphere bundle projection to the base manifold descends to a continuous surjective map from the reduced space at zero to the orbit quotient of the configuration space, but it fails to be a morphism of stratified spaces if we endow the reduced space with its contact stratification and the base space with the customary orbit type stratification defined by the Lie group action. The present paper introduces a new stratification of the contact quotient at zero, called in what follows the $C$ - $L$ stratification (standing for the coisotropic or Legendrian nature of its pieces), which solves the above-mentioned two problems. Its main features are the following. First, it is compatible with the contact stratification of the quotient and the orbit type stratification of the configuration orbit space. It is also finer than the contact stratification. Unlike the cotangent bundle case, the isotropy lattice of the group action on the base manifold $Q$ no longer suffices for the description of this new stratification. In fact, this lattice $I_{Q}$ indexes a new decomposition of each contact stratum of the reduced space, but the isotropy lattice of the zero level set of the momentum map is given by $I_{Q}$ without those elements corresponding to orbit type submanifolds of dimension equal to that of their orbits. Second, the natural projection of the C-L stratified quotient space to its base space, stratified by orbit types, is a morphism of stratified spaces. Third, each C-L stratum is a bundle over an orbit type stratum of the base and each contact stratum can be seen as a union of C-L pieces, one of them being open and dense in its corresponding contact stratum and contactomorphic to a cosphere bundle. The other strata are coisotropic or Legendrian submanifolds in the contact components that contain them.

The paper is structured as follows. Section 2 presents the definitions, conventions, and results on stratified spaces and contact reduction (regular and singular) that are used throughout the paper. Section 3 quickly reviews the relevant results on regular contact cosphere reduction. Section 4 presents the stratification of the zero level set of the momentum map and begins the work on the stratification of the quotient by studying the case of one single orbit type (Theorem 4.1). The contact stratification and contact geometry of the reduced space are studied in Section 5, having as main results Theorems 5.1 and 5.2. The new C-L stratification is also introduced here and its properties are investigated. Theorem 5.3 presents a complete description of its frontier conditions. Also explained is the tool needed for an 
analysis of Whitney or local triviality conditions for this new stratification. Section 6 studies the singular cosphere bundle reduction for almost semifree actions, that is, actions that are in bijective correspondence with free lifted actions on the cosphere bundle. The stratification is computed explicitly, and the particular case of the circle acting on the cosphere bundle of the plane is carried out in detail. Section 7 studies the example of the diagonal action of the two-torus on two copies of the plane, lifted to the cosphere bundle. This example is rich enough to illustrate the relationships between the various stratifications, and the strata are computed explicitly.

\section{Preliminaries}

In this section we will survey the main results of several topics that will be needed in the subsequent development of the paper. We will assume that all topological spaces are paracompact. In addition, manifolds will be real, smooth and finitedimensional. By a group we will mean a finite-dimensional Lie group. Every action of a group $G$ on a manifold $M$ is supposed to be smooth, and the usual notation $g \cdot m$ for $g \in G$ and $m \in M$ will be employed. The natural pairing between a vector space and its dual will be denoted by $\langle\cdot, \cdot\rangle$. By a submanifold, we will always mean an embedded submanifold.

2.1. Stratified spaces and proper group actions. The natural framework for singular reduction is the category of stratified spaces. We briefly recall here the basic concepts (see [18]). Let $X$ be a topological space and $\mathcal{Z}_{X}=\left\{S_{i}: i \in I\right\}$ a locally finite partition of $X$ into locally closed disjoint subspaces $S_{i} \subset X$, where $I$ is some index set. We say that $\left(X, \mathcal{Z}_{X}\right)$ is a decomposed space if every $S_{i}$ is a manifold whose topology coincides with the induced one from $X$ and if the frontier condition holds: $S_{i} \cap \overline{S_{j}} \neq \varnothing$ implies $S_{i} \subset \overline{S_{j}}$, whence $S_{i} \subset \partial S_{j}$, where $\partial S_{j}:=\overline{S_{j}} \backslash S_{j}$. In this case, the elements of $\mathcal{Z}_{X}$ are called pieces of the decomposition.

In a topological space $X$, two subsets $A$ and $B$ are said to be equivalent at $x$ if there exists an open neighborhood $U$ of $x$ such that $A \cap U=B \cap U$. These equivalence classes are called set germs at $x$. Let $\mathcal{S}$ be the map that associates to each point $x \in X$ the set germ $\mathcal{S}_{x}=[O]_{x}$ of a locally closed subset $O$ of $X$. We say that $(X, \mathcal{S})$ is a stratified space if, for every point $x \in X$ there exists a neighborhood $U$ of $x$ endowed with a decomposition $\mathcal{Z}_{U}$ such that for every $y \in U$, $\mathcal{S}_{y}=[Z(y)]_{y}$, where $Z(y) \in \mathcal{Z}_{U}$ denotes the piece containing $y$. In this case we say that the decomposition $\mathcal{Z}_{U}$ locally induces $\mathcal{S}$.

Given two stratified spaces $(X, \mathcal{S})$ and $(Y, \mathcal{T})$ and a continuous map $f: X \rightarrow Y$, we say that $f$ is a morphism of stratified spaces (or shorter, a morphism) if for every $x \in X$ there exist neighborhoods $V$ of $f(x)$ and $U \subset f^{-1}(V)$ of $x$ such that

(i) there exist decompositions $\mathcal{Z}_{U}$ and $\mathcal{Z}_{V}$ locally inducing the stratifications $\mathcal{S}$ and $\mathcal{T}$ respectively, with the property that for every $y \in U$ contained in a piece $S \in \mathcal{Z}_{U}$ there is an open neighborhood $y \in W \subset U$ such that $\left.f\right|_{W}(S \cap W)$ is contained in the unique piece $R \in \mathcal{Z}_{V}$ that contains $f(y)$, and

(ii) $\left.f\right|_{S \cap W}: S \cap W \rightarrow R$ is smooth.

In addition, we will say that $f$ is a stratified immersion (resp. submersion, diffeomorphism, etc.) if so are all the maps $\left.f\right|_{S \cap W}$ for every point $x \in X$. Given two different stratifications $\mathcal{S}$ and $\mathcal{S}^{\prime}$ on the same topological space $X$, we say that $\mathcal{S}$ 
is finer than $\mathcal{S}^{\prime}$ if the identity map $1_{X}$, viewed as a map between stratified spaces $(X, \mathcal{S}) \rightarrow\left(X, \mathcal{S}^{\prime}\right)$, is a morphism.

Smooth manifolds are trivially stratified spaces, and smooth maps between manifolds are their morphisms. Note that a decomposed space $\left(X, \mathcal{Z}_{X}\right)$ induces naturally a stratification $(X, \mathcal{S})$ by just taking $S_{x}$ to be the set germ of the piece containing $x$, for every $x \in X$. In this case, we call the pieces $S_{i} \in \mathcal{Z}_{X}$ the strata of $(X, \mathcal{S})$ and say that they satisfy the frontier conditions defined by the underlying decomposition. In this paper the stratifications that will appear will be of this form and thus, for the sake of simplicity, when this is the case we will work most of the time with the decompositions inducing these stratifications.

Let $\phi: G \times M \rightarrow M$ be a smooth action of the Lie group $G$ on the manifold $M$. Since $M$ is paracompact it admits a Riemannian metric; so if it is connected, $M$ is second countable. The action is called proper if $\phi \times \mathrm{id}_{M}$ is a proper map. In this paper we only work with proper actions. For instance, every action of a compact group is automatically proper. The main properties of a proper action of $G$ on $M$ are:

(i) For each $m \in M$, its stabilizer (or isotropy group) $G_{m}$ is compact.

(ii) The manifold structure of the orbit $G \cdot m$ is the one that makes the natural bijection $G / G_{m} \rightarrow G \cdot m$ a diffeomorphism. The inclusion $G \cdot m \hookrightarrow M$ is an injective immersion. In addition, the orbit is a closed subset of $M$. If $M$ is connected, then the orbit is an embedded submanifold of $M$.

(iii) The quotient space equipped with the quotient topology is paracompact and the orbit map $\pi: M \rightarrow M / G$ is open and closed.

(iv) $M$ admits a $G$-invariant Riemannian metric.

(v) If all the stabilizer groups are conjugate to a given subgroup $H \subset G$, then $M / G$ is a smooth manifold, the orbit map $\pi: M \rightarrow M / G$ is a smooth locally trivial fiber bundle whose fibers are diffeomorphic to $G / H$, and the structure group of this locally trivial fiber bundle is $N(H) / H$, where $N(H)$ is the normalizer of $H$ in $G$.

We now quote Palais' Tube Theorem [16] in a form adapted to our needs, which is of great importance in the local study of proper actions. Let $m \in M$. Choose an invariant Riemannian metric on $M$ and use it to decompose $T_{m} M=\mathfrak{g} \cdot m \oplus S_{m}$, where $\mathfrak{g} \cdot m=\left\{\xi_{M}(m): \xi \in \mathfrak{g}\right\}$. This splitting is $G_{m}$-invariant for the linear action of $G_{m}$ on $T_{m} M$. The twisted action of $G_{m}$ on $G \times S_{m}$ is defined by

$$
h \cdot(g, s)=\left(g h^{-1}, h \cdot s\right)
$$

for $h \in G_{m}, g \in G$ and $s \in S_{m}$. Since $G_{m}$ acts freely on the right on $G$, the twisted action is free. In addition, $G_{m}$ is compact by property (i) of proper group actions, so the quotient space, denoted by $G \times_{G_{m}} S_{m}$, is a manifold. The Tube Theorem implies the existence of a $G_{m}$-invariant open ball $U$ around the origin in $S_{m}$ such that the map $\psi: G \times_{G_{m}} S_{m} \rightarrow M$ defined by

$$
\psi([g, s])=g \cdot \exp _{m}(s)
$$

maps $G \times \times_{G_{m}} U$ diffeomorphically and equivariantly onto a $G$-invariant neighborhood $U^{\prime}$ of $G \cdot m$ in $M$. Here, $\exp _{m}$ is the exponential map at $m$ associated with the chosen Riemannian metric. The map $\psi$ is called a tube for the action and $S_{m}$ is called a linear slice, or simply a slice of the action at $m$. 
Let $I_{M}$ be the isotropy lattice of $M$, i.e., the set of conjugacy classes of subgroups of $G$ which appear as stabilizers for the action of $G$ on $M$. Such classes, called orbit types, are denoted by $(H)$. For each element $(H) \in I_{M}$ the $(H)$-orbit type manifold is defined by

$$
M_{(H)}=\left\{m \in M \mid\left(G_{m}\right)=(H)\right\} .
$$

In the same way, for any subset $A$ of $M$ one defines the orbit type sets of $A$ by $A_{(H)}=A \cap M_{(H)}$ and the isotropy lattice of $A$ by restriction. For a proper $G$-action on a manifold $M$ such that $M / G$ is connected, there is always a subgroup $H_{0} \subset G$ such that $M_{\left(H_{0}\right)}$ is open and dense in $M$ and $H_{0}$ is conjugate to a proper subgroup of any other stabilizer. This orbit type $\left(H_{0}\right)$ is called the principal orbit type of $I_{M}$.

Obviously, the collection of orbit type manifolds forms a partition of $M$. For simplicity, we will from now on make the following important assumption: for every $(H) \in I_{M}$, all the connected components of $M_{(H)}$ have the same dimension and $M$ is second countable. Hence we have:

(i) For every $(H) \in I_{M}, M_{(H)}$ is a $G$-invariant submanifold of $M$, and

(ii) $M$ and $M / G$ are stratified spaces with strata $M_{(H)}$ and $M^{(H)}:=M_{(H)} / G$ respectively. Their frontier conditions are:

$$
M^{(H)} \subset \partial M^{(L)} \Longleftrightarrow(L) \prec(H),
$$

and correspondingly for $M$, where $(L) \prec(H)$ means that $L$ is conjugate to a proper subgroup of $H$. Since $\prec$ defines a partial ordering in $I_{M}$ we say that the frontier conditions of the stratification of $M / G$ are induced by the isotropy lattice $I_{M}$.

Remark 2.1. If one allows the connected components of the orbit type manifolds to have different dimensions, then one needs to work in the larger category of $\Sigma$ manifolds and $\Sigma$-decompositions. A $\Sigma$-manifold is a countable topological sum of connected smooth manifolds having possibly different dimensions (see [18 for more details). However, our results on the stratified nature of the studied quotient spaces remain valid.

2.2. Reduction of contact manifolds. Recall that a contact structure on a smooth $(2 n+1)$-dimensional manifold $\mathcal{C}$ is a codimension one smooth distribution $\mathcal{H} \subset T C$ maximally non-integrable in the sense that it is locally given by the kernel of a one-form $\eta$ with $\eta \wedge(d \eta)^{n} \neq 0$. Such an $\eta$ is called a (local) contact form. Any two proportional contact forms define the same contact structure. A contact structure which is the kernel of a global contact form is called exact. In the case of exact contact manifolds, $d \eta$ has rank $n$, implying the existence of the Reeb vector field $R$ uniquely defined by

$$
i_{R} d \eta=0 \quad \text { and } \quad \eta(R)=1 .
$$

In the following we will consider only exact orientable contact manifolds.

When studying the geometry of the singular reduced spaces of cosphere bundles, one needs the notions of coisotropic and isotropic submanifolds in the contact context. Any integral submanifold $N$ of $\mathcal{H}$ has the property that its tangent space at every point is an isotropic subspace of the symplectic vector space $\left(\right.$ ker $\eta_{x}, d \eta_{x}$ ) and that's why, sometimes, they are also called isotropic submanifolds. In particular, 
$\operatorname{dim} N \leq n$; if $\operatorname{dim} N=n$, then $N$ is called a Legendrian submanifold. A submanifold $N$ of the contact manifold $(\mathcal{C}, \eta, R)$ is coisotropic if for any $x \in N$ the subspace $T_{x} N \cap \operatorname{ker} \eta_{x}$ is coisotropic in the symplectic vector space (ker $\eta_{x}, d \eta_{x}$ ).

A group $G$ is said to act by contactomorphisms on a contact manifold if it preserves the contact structure $\mathcal{H}$. For an exact contact manifold $(\mathcal{C}, \eta)$, this means that $g^{*} \eta=f_{g} \eta$ for a smooth, real-valued, nowhere zero function $f_{g}$. $G$ acts by strong contactomorphisms on $\mathcal{C}$, if $g^{*} \eta=\eta$; i.e., $G$ preserves the contact form, not only the contact structure. A $G$-action by strong contactomorphisms on $(\mathcal{C}, \eta)$ admits an equivariant momentum map $J: N \rightarrow \mathfrak{g}^{*}$ given by evaluating the contact form on the infinitesimal generators of the action: $\langle J(x), \xi\rangle:=\eta\left(\xi_{\mathcal{C}}\right)(x)$. Note the main difference with respect to the symplectic case: any action by strong contactomorphisms is automatically Hamiltonian. Note also that orbits which lie in the zero level set of the contact momentum map are examples of isotropic submanifolds. For more details on contact manifolds and their associated momentum maps see [2], 9], and 20].

Reduction theory for co-oriented contact manifolds in the singular context was introduced by Willett in [20]. We now review briefly this construction at zero momentum, since it will be used in our next refinement to the cosphere bundle case. Let $G$ be a group that acts by strong contactomorphisms on an exact contact manifold $(\mathcal{C}, \eta)$. By the definition of the momentum map, its zero level set is a $G$-space. The contact quotient (reduced space) of $\mathcal{C}$ at zero momentum is defined as

$$
\mathcal{C}_{0}:=J^{-1}(0) / G
$$

Note that, as in the symplectic case, this quotient is in general a singular space.

Theorem 2.1. Let $(\mathcal{C}, \eta)$ be an exact contact manifold and $G$ a Lie group acting properly on $\mathcal{C}$ by strong contactomorphisms. Then for every stabilizer subgroup $H$ of $G$ the set

$$
\mathcal{C}_{0}^{(H)}:=\left(J^{-1}(0)\right)_{(H)} / G=\left(\mathcal{C}_{(H)} \cap J^{-1}(0)\right) / G
$$

is a smooth manifold, and the partition of the contact quotient

$$
\mathcal{C}_{0}:=\left(J^{-1}(0)\right) / G
$$

into these manifolds is a stratification with frontier condition induced by the partial order of $I_{J^{-1}(0)}$. Moreover, there is a reduced exact contact structure on $\mathcal{C}_{0}^{(H)}$ generated by the one-form $\eta_{0}^{(H)}$ characterized by

$$
\pi_{G}^{(H)} \eta_{0}^{(H)}=\tilde{i}_{(H)} \eta,
$$

where $\pi_{G}^{(H)}:\left(J^{-1}(0)\right)_{(H)} \rightarrow \mathcal{C}_{0}^{(H)}$ is the projection on the orbit space and $\tilde{i}_{(H)}$ : $\left(J^{-1}(0)\right)_{(H)} \hookrightarrow \mathcal{C}$ is the inclusion.

In what follows this stratification will be referred to as the contact stratification of $\mathcal{C}_{0}$.

\section{Regular Cosphere Bundle Reduction}

Cosphere bundles are the odd-dimensional analogs of cotangent bundles in contact geometry. In the following, we will briefly recall their construction and their equivariant regular contact reduction, referring to [5] and [19] for more details. 
Let $Q$ be an $n$-dimensional manifold and $\theta$ the Liouville one-form on $T^{*} Q$, defined by $\theta\left(X_{p_{x}}\right)=\left\langle p_{x}, T_{p_{x}} \tau X_{p_{x}}\right\rangle$, where $p_{x} \in T_{x}^{*} Q, X \in T_{p_{x}}\left(T^{*} Q\right)$, and $\tau: T^{*} Q \rightarrow Q$ is the canonical projection. Let $\Phi: G \times Q \rightarrow Q$ be an action of $G$ on $Q$. Denote by

$$
\Phi_{*}: G \times T^{*} Q \rightarrow T^{*} Q
$$

its natural (left) lift to the cotangent bundle. Consider the action of the multiplicative group $\mathbb{R}_{+}$by dilations on the fibers of $T^{*} Q \backslash\left\{0_{T^{*} Q}\right\}$.

Definition 3.1. The cosphere bundle $S^{*} Q$ of $Q$ is the quotient manifold $\left(T^{*} Q \backslash\left\{0_{T^{*} Q}\right\}\right) / \mathbb{R}_{+}$.

Let $\pi_{+}: T^{*} Q \backslash\left\{0_{T^{*} Q}\right\} \rightarrow S^{*} Q$ and $\kappa:\left[\alpha_{q}\right] \in S^{*} Q \mapsto q \in Q$ be the canonical projections. Denote by $\left[\alpha_{q}\right]$ the elements of the cosphere bundle. Of course, $\left(\pi_{+}, \mathbb{R}_{+}, T^{*} Q \backslash\left\{0_{T^{*} Q}\right\}, S^{*} Q\right)$ is an $\mathbb{R}_{+}$-principal bundle. Also, we will use the $\pi_{+}$ notation for any $\mathbb{R}_{+}$projection. The exact contact structure of $S^{*} Q$ is given by the kernel of any one-form $\theta_{\sigma}$ satisfying $\theta_{\sigma}=\sigma^{*} \theta$ for $\sigma: S^{*} Q \rightarrow T^{*} Q \backslash\left\{0_{T^{*} Q}\right\}$ a global section. Such a $\sigma$ always exists and, even more, the set of global sections of this principal bundle is in bijective correspondence with the set of $C^{\infty}$ functions $f: T^{*} Q \backslash\left\{0_{T^{*} Q}\right\} \rightarrow \mathbb{R}_{+}$satisfying

$$
f_{\sigma}\left(r \alpha_{q}\right)=\frac{1}{r} f_{\sigma}\left(\alpha_{q}\right), \quad r \in \mathbb{R}_{+}, \alpha_{q} \in T^{*} Q \backslash\left\{0_{T^{*} Q}\right\} .
$$

(See [5] for details.)

Remark 3.1. 1. Let $\mathcal{C}\left(S^{*} Q\right)=S^{*} Q \times \mathbb{R}_{+}$be the symplectic cone over $S^{*} Q$, endowed with the symplectic form $d\left(t \theta_{\sigma}\right)$. Then one can easily see that $T_{\sigma}: \mathcal{C}\left(S^{*} Q\right) \rightarrow T^{*} Q$ given by $T_{\sigma}\left(\left[\alpha_{q}\right], t\right)=t f_{\sigma}\left(\alpha_{q}\right) \alpha_{q}$ is a well-defined symplectic diffeomorphism, that is, a symplectomorphism.

2. If $Q$ is zero-dimensional, we set, by convention, $S^{*} Q=\varnothing$.

The action $\Phi$ lifts to the cosphere bundle yielding a proper action

$$
\widehat{\Phi}_{*}: G \times S^{*} Q \rightarrow S^{*} Q, \quad \widehat{\Phi}_{*}\left(g,\left[\alpha_{q}\right]\right)=\left[\Phi_{*}\left(g, \alpha_{q}\right)\right]
$$

by contactomorphisms with all scale factors positive. In [1] it has been proved that for any proper action which preserves an exact contact structure, there exists a $G$ invariant contact form. As every contact form on the cosphere bundle is obtained via a global section as above, we shall choose once and for all a section $\sigma$ for which $\left(\widehat{\Phi}_{* g}\right)^{*} \theta_{\sigma}=\theta_{\sigma}$. Relative to this contact form the induced action on the cosphere bundle is by strong contactomorphisms. The associated momentum map, which depends on the section $\sigma$, will be denoted $J$ for simplicity, since in what follows no other contact form different from $\theta_{\sigma}$ will be used. As above, the exact contact structure of $S^{*}(Q / G)$ can be described as the kernel of a global contact form of type $\Theta_{\Sigma}$, where

$$
\Sigma: S^{*}(Q / G) \rightarrow T^{*}(Q / G) \backslash\left\{0_{T^{*}(Q / G)}\right\}
$$

is a global section, and $\Theta$ is the Liouville one-form of $T^{*}(Q / G)$.

Regular reduction of cosphere bundles was done in [5]. Its main result at zero momentum is

Theorem 3.1. Let $G$ be a finite-dimensional Lie group acting freely and properly on a differentiable manifold $Q$. Then $\left(S^{*} Q\right)_{0}$, the reduced space at the regular value zero of the cosphere bundle of $Q$, is contact diffeomorphic to the cosphere bundle $S^{*}(Q / G)$. 
In the remainder of this paper, we will generalize this result to non-free actions, within the framework of stratified spaces, relating our results to the contact stratification defined in Theorem 2.1.

\section{The DeComposition of $J^{-1}(0)$}

The geometric study of the contact reduced space $\left(S^{*} Q\right)_{0}$ passes through the analysis of the level set $J^{-1}(0)$ and, in particular, of its isotropy lattice $I_{J^{-1}(0)}$. We shall use the fact that both the cosphere bundle $S^{*} Q$ and the lifted action of $G$ on it are completely determined by the differential structure of $Q$ and its supported $G$-action. This will allow us to obtain our first main result, Proposition 4.1. which describes this isotropy lattice, and hence the topology of the contact stratification of $\left(S^{*} Q\right)_{0}$, in terms of the isotropy lattice of $Q$ without those elements corresponding to zero-dimensional orbit types in $Q / G$. Also, as a preliminary result, and a "building block" for the general construction, we state an intermediary cosphere reduction result, Theorem 4.1, which applies to base manifolds $Q$ on which the group action is not free but exhibits a single orbit type; that is, $I_{Q}$ consists of only one element.

Lemma 4.1. The isotropy lattice of the cosphere bundle coincides with the isotropy lattice of the cotangent bundle without the zero section

$$
I_{S^{*} Q}=I_{T^{*} Q \backslash\left\{0_{T^{*} Q}\right\}} \cdot
$$

Proof. It is enough to show that $G_{\alpha_{q}}=G_{\left[\alpha_{q}\right]}$ for any $\alpha_{q} \in T^{*} Q \backslash\left\{0_{T^{*} Q}\right\}$. Thus let $g \in G_{\left[\alpha_{q}\right]}$. This implies that $g\left[\alpha_{q}\right]=\left[g \alpha_{q}\right]=\left[\alpha_{q}\right] \Longleftrightarrow g \alpha_{q}=r \alpha_{q}$ for $r>0$. Since the action of $G$ on $Q$ is proper, there is a $G$-invariant Riemannian metric on $Q$ and hence $\left\|g \alpha_{q}\right\|=\left\|\alpha_{q}\right\|=r\left\|\alpha_{q}\right\|$. It follows that $r=1$ and $G_{\left[\alpha_{q}\right]} \subset G_{\alpha_{q}}$. The other inclusion being obvious, the proof is now complete.

Remark 4.1. We will write $J_{c t}: T^{*} Q \rightarrow \mathfrak{g}^{*}$ for the canonical momentum map for the cotangent-lifted action of $G$ on $T^{*} Q$ endowed with the canonical symplectic form. As $J^{-1}(0)=\pi_{+}\left(J_{c t}^{-1}(0) \backslash\left\{0_{T^{*} Q}\right\}\right)$ note that

$$
\left(J^{-1}(0)\right)_{(L)}=\pi_{+}\left(\left(J_{c t}^{-1}(0)\right)_{(L)} \backslash\left[\left(J_{c t}^{-1}(0)\right)_{(L)} \cap\left\{0_{T^{*} Q}\right\}\right]\right)
$$

since

$$
\left(J_{c t}^{-1}(0) \backslash\left\{0_{T^{*} Q}\right\}\right)_{(L)}=\left(J_{c t}^{-1}(0)\right)_{(L)} \backslash\left[\left(J_{c t}^{-1}(0)\right)_{(L)} \cap\left\{0_{T^{*} Q}\right\}\right] .
$$

The following theorem is an immediate consequence of Theorems 3.1] and 2.1]

Theorem 4.1. Let $G$ be a finite-dimensional Lie group acting properly on the differentiable manifold $Q$ such that all the points in $Q$ have stabilizers conjugate to some $K$ (that is, $\left.Q=Q_{(K)}\right)$. Then $J^{-1}(0)$ is a submanifold of $\left(S^{*} Q\right)_{(K)}$ and $\left(S^{*} Q\right)_{0}$, the reduced space at zero, is contact-diffeomorphic to $S^{*}(Q / G)$.

In the following proposition we give the decomposition of $J^{-1}(0)$ and show how the topology of the contact quotient at zero is completely determined by the isotropy lattice of $Q$. For that, we will use the following partition of $T^{*} Q$. We fix once and for all a $G$-invariant Riemannian metric on $Q$. Then, for any $(H) \in I_{Q}$, the restriction of $T Q$ to the submanifold $Q_{(H)}$ can be decomposed as the Whitney sum $T_{Q_{(H)}} Q=T Q_{(H)} \oplus N Q_{(H)}$, where, for every $q \in Q_{(H)}, N_{q} Q_{(H)}=T_{q} Q_{(H))}^{\perp}$. Note that each of the elements of the Whitney sum are $G$-invariant vector bundles over 
$Q_{(H)}$. Dualizing this splitting over each orbit type submanifold in $Q$, we obtain the following $G$-invariant partition of $T^{*} Q$ :

$$
T^{*} Q=\coprod_{(H) \in I_{Q}} T^{*} Q_{(H)} \oplus N^{*} Q_{(H)} .
$$

Now, the restriction of this partition to $T^{*} Q \backslash\left\{0_{T^{*} Q}\right\}$, and afterwards its quotient by the action of $\mathbb{R}_{+}$, induces a $G$-invariant partition of $S^{*} Q$.

Let $I_{Q}^{*}$ denote the isotropy lattice of $Q$ without those elements $(H)$ corresponding to orbit type submanifolds $Q_{(H)}$ for which the orbits of the restricted $G$-action have the same dimension as $Q_{(H)}$. At this moment, we will need some results on cotangent-lifted actions, which were proved in [17.

Lemma 4.2. Assume that $G$ acts on $Q$ and on $T^{*} Q$ by cotangent lifts with momentum map $J_{c t}: T^{*} Q \rightarrow \mathfrak{g}^{*}$. Let $(L),(H) \in I_{Q}$ be arbitrary.

(i) $\left(N^{*} Q_{(H)}\right)_{(H)}$ is the zero section of $N^{*} Q_{(H)}$.

(ii) Let $J_{c t(H)}$ denote the canonical momentum map on $T^{*} Q_{(H)}$ associated with the lift of the action on $Q_{(H)}$ obtained by restriction from $Q$. Then

$$
\left(J_{c t}^{-1}(0)\right)_{(L)}=J_{c t(L)}^{-1}(0) \coprod_{(H) \succ(L)}\left(J_{c t(H)}^{-1}(0) \times\left(N^{*} Q_{(H)}\right)_{(L)}\right) .
$$

(iii) If $(L) \neq(H)$, then $\left(N^{*} Q_{(H)}\right)_{(L)} \neq \varnothing$ if and only if $(H) \succ(L)$.

Proposition 4.1. Suppose $G$ acts properly on the manifold $Q$. Then we have:

(i) For $q \in Q_{(H)}$ such that $G_{q}=H$ and $(L) \in I_{S^{*} Q}$,

$$
\left(J^{-1}(0)\right)_{(L)} \cap S_{q}^{*} Q \neq \varnothing \Longleftrightarrow(L) \in I_{Q} \text { and }\left((H) \in I_{Q}^{*} \text { or }(L) \prec(H)\right) \text {. }
$$

(ii) $(L) \in I_{J^{-1}(0)} \Longleftrightarrow(L) \in I_{Q}^{*}$ and hence $\mathcal{C}_{0}^{(L)} \neq \varnothing \Longleftrightarrow(L) \in I_{Q}^{*} \Longleftrightarrow$ $\operatorname{dim} Q^{(L)} \geq 1$.

(iii) The cosphere bundle projection $k$ restricts to the $G$-equivariant continuous surjection $k_{(L)}:\left(J^{-1}(0)\right)_{(L)} \rightarrow \overline{Q_{(L)}}$ which is also an open map.

(iv) For a fixed orbit type $(L)$ in the zero momentum level set of the lifted $G$ action to $S^{*} Q$ the corresponding orbit type submanifold admits the following $G$-invariant partition:

$$
\left(J^{-1}(0)\right)_{(L)}=J_{(L)}^{-1}(0) \coprod_{(H) \succ(L)} \pi_{+}\left(J_{c t(H)}^{-1}(0) \times\left(N^{*} Q_{(H)}\right)_{(L)}\right),
$$

where $(H) \in I_{Q}$.

(v) For every $(H) \succ(L)$ with $(L) \in I_{Q}^{*}$ and $(H) \in I_{Q}$ the restrictions

$\tilde{t}_{(L)}:=\left.k_{(L)}\right|_{J_{(L)}^{-1}(0)} \quad$ and $\quad \tilde{t}_{(H) \succ(L)}:=\left.k_{(L)}\right|_{\pi_{+}\left(J_{c t(H)}^{-1}(0) \times\left(N^{*} Q_{(H)}\right)_{(L)}\right)}$

are $G$-equivariant smooth surjective submersions onto $Q_{(L)}$ and $Q_{(H)}$ respectively. The mappings $J_{c t(H)}$ and $J_{(H)}$ denote the momentum maps of the restricted actions of $G$ to $T^{*} Q_{(H)}$ and $S^{*} Q_{(H)}$ respectively (which are the same as the canonical momentum maps for the restricted $G$-action on $\left.Q_{(H)}\right)$.

Proof. To prove (i), let $(L) \in I_{S^{*} Q}$ and $q \in Q_{(H)}$ with $G_{q}=H$. Then

$$
\left(J_{c t}^{-1}(0)\right)_{(L)} \cap T_{q}^{*} Q=\left(S_{q}^{H}\right)^{*} \oplus\left(N_{q}^{*} Q_{(H)}\right)_{(L)},
$$


where $S_{q}^{H}$ is the linear slice for the $G$-action on $Q_{(H)}$ (see section 3 of [17]). Since $\left(J^{-1}(0)\right)_{(L)} \cap S_{q}^{*} Q=\varnothing \Longleftrightarrow\left(J_{c t}^{-1}(0)\right)_{(L)} \cap T_{q}^{*} Q=\{0\}$, then $\left(J^{-1}(0)\right)_{(L)} \cap S_{q}^{*} Q=$ $\varnothing$ only when $\left(S_{q}^{H}\right)^{*}$ and $\left(N_{q}^{*} Q_{(H)}\right)_{(L)}$ are simultaneously zero. This amounts to $(L)=(H) \in I_{Q} \backslash I_{Q}^{*}$, (see Lemma 4.2) from which the result follows.

(ii) is a forward consequence of (i). The rest of this statement and the $G$ equivariant continuous surjectivity of $k_{(L)}$ are direct consequences of the fact that $I_{Q}=I_{J_{c t}^{-1}(0)}$. To prove the openness of $k_{(L)}$ it suffices to observe that for any open subset $\mathcal{U}$ of $\left(J^{-1}(0)\right)_{(L)}, k_{(L)}(\mathcal{U})=\tau_{(L)}\left(\pi^{-1}(\mathcal{U})\right)$, where $\tau_{(L)}:\left(J_{c t}^{-1}(0)\right)_{(L)} \rightarrow \overline{Q_{(L)}}$ is the open canonical cotangent projection map.

Applying (4.1) and the fact that $\left(N^{*} Q_{(H)}\right)_{(L)}$ does not contain the zero section when $(H) \neq(L)$ we have

$$
\left(J_{c t}^{-1}(0) \backslash\left\{0_{T^{*} Q}\right\}\right)_{(L)}=\left(J_{c t(L)}^{-1}(0)\right) \backslash\left\{0_{T^{*} Q_{(L)}}\right\} \coprod_{(H) \succ(L)}\left[J_{c t(H)}^{-1}(0) \times\left(N^{*} Q_{(H)}\right)_{(L)}\right] .
$$

Hence, applying $\pi_{+}$to this relation, we get

$$
\left(J^{-1}(0)\right)_{(L)}=J_{(L)}^{-1}(0) \coprod_{(H) \succ(L)} \pi_{+}\left(J_{c t(H)}^{-1}(0) \times\left(N^{*} Q_{(H)}\right)_{(L)}\right),
$$

which proves statement (iv).

As for the proof of (v), it is enough to notice that

$$
J_{(L)}^{-1}(0) \quad \text { and } \quad \pi_{+}\left(J_{c t(H)}^{-1}(0) \times\left(N^{*} Q_{(H)}\right)_{(L)}\right)
$$

are bundles over $Q_{(L)}$ and $Q_{(H)}$ respectively.

Remark 4.2. Notice that for the description of orbit types in $J^{-1}(0)$, we need not only $I_{Q}^{*}$, but also the lattice $I_{Q}$ since each $\left(J^{-1}(0)\right)_{(L)}$ is written as a union with index $(H)$ in $I_{Q}$, but $(L)$ belongs to $I_{Q}^{*}$.

\section{TOPOlOGy AND CONTACT GeOMETRY OF $\mathcal{C}_{0}$}

5.1. The secondary decomposition of $\mathcal{C}_{0}^{(L)}$. Define the fiber bundles:

$$
\begin{gathered}
s_{(H) \succ(L)}:=J_{c t(H)}^{-1}(0) \times\left(N^{*} Q_{(H)}\right)_{(L)} \rightarrow Q_{(H)}, \\
s_{(L)}:=J_{c t(L)}^{-1}(0) \rightarrow Q_{(L)} .
\end{gathered}
$$

Taking into account that $\pi_{+}\left(s_{(H) \succ(L)}\right)$ are $G$-invariant pieces of the partition (4.2) of $\left(J^{-1}(0)\right)_{(L)}$ and that the actions of $G$ and $\mathbb{R}_{+}$commute, we can define:

$$
\begin{gathered}
C S_{(H) \succ(L)}:=\frac{\pi_{+}\left(s_{(H) \succ(L)}\right)}{G}, \\
C C_{(L)}:=\frac{J_{(L)}^{-1}(0)}{G}=\frac{\pi_{+}\left(s_{(L)} \backslash\left\{0_{T^{*} Q_{(L)}}\right\}\right)}{G} \simeq S^{*}\left(\frac{Q_{(L)}}{G}\right) .
\end{gathered}
$$


Notice that for the above equivalence we have applied Theorem 4.1 and that each contact stratum admits the following partition, which is the quotient of (4.2):

$$
\begin{aligned}
\mathcal{C}_{0}^{(L)} & =\frac{\left(J^{-1}(0)\right)_{(L)}}{G}=C C_{(L)} \coprod_{(H) \succ(L)} C S_{(H) \succ(L)} \\
& \simeq S^{*}\left(\frac{Q_{(L)}}{G}\right) \coprod_{(H) \succ(L)} C S_{(H) \succ(L)} .
\end{aligned}
$$

Remark 5.1. In the notation of the previous section, the maps $k_{(L)}, \widetilde{t}_{(L)}$, and $\widetilde{t}_{(H) \succ(L)}$ descend to

$$
k^{(L)}: \mathcal{C}_{0}^{(L)} \rightarrow \overline{Q^{(L)}}, \quad \widetilde{t}^{(L)}: C C_{(L)} \rightarrow Q^{(L)} \text {, and } \quad \widetilde{t}^{(H) \succ(L)}: C S_{(H) \succ(L)} \rightarrow Q^{(H)} ;
$$

$k^{(L)}$ is an open continuous surjection, and the other two are smooth surjective submersions.

Theorem 5.1. With the above notation, we obtain the following:

(i) $\overline{Q^{(L)}}$ is a stratified space with strata $Q^{(H)}$, for all $(L) \preccurlyeq(H)$ and with frontier conditions given by

$$
Q^{(K)} \cap \overline{Q^{(H)}} \neq \varnothing \Longleftrightarrow(H) \preccurlyeq(K) .
$$

Moreover, $Q^{(L)}$ is open and dense in $\overline{Q^{(L)}}$.

(ii) For every $(L) \in I_{Q}^{*}$ and $(H) \in I_{Q}$, the partition (5.1) is a stratification of the corresponding contact stratum $\mathcal{C}_{0}^{(L)}$, called the secondary stratification. The frontier conditions are given by

$$
\begin{aligned}
& C S_{(H) \succ(L)} \subset \partial C C_{(L)} \text { for all }(H) \succ(L) ; \\
& C S_{\left(H^{\prime}\right) \succ(L)} \subset \partial C S_{(H) \succ(L)} \Longleftrightarrow\left(H^{\prime}\right) \succ(H) \succ(L) \text {. }
\end{aligned}
$$

Moreover, the piece $C C_{(L)}$ is diffeomorphic to $S^{*} Q^{(L)}$, is open and dense in $\mathcal{C}_{0}^{(L)}$, and the map $k^{(L)}$ is a surjective submersion of stratified spaces.

Proof. Since the $G$-action is proper, the orbit type decomposition of $Q$ induces a stratification of $Q / G$ and the first part of the theorem follows immediately considering the relative topology of $\overline{Q^{(L)}}$ in $Q / G$. Also, (5.1) is a locally finite partition and its pieces are obviously submanifolds of $\mathcal{C}_{0}^{(L)}$. As $k^{(L)}$ is a continuous map and $\left(k^{(L)}\right)^{-1}\left(Q^{(L)}\right)=C C_{(L)}$, it follows that $C C_{(L)}$ is open in $\mathcal{C}_{0}^{(L)}$. In order to prove the density, let $x \in \mathcal{C}_{0}^{(L)}$ and $\mathcal{U}$ be any open neighborhood of $x$. Hence, $\mathcal{V}=k^{(L)}(\mathcal{U})$ is an open subset of $\overline{Q^{(L)}}$ and, since $Q^{(L)}$ is dense in $\overline{Q^{(L)}}$, there is at least one element $y \in \mathcal{V} \cap Q^{(L)}$. Notice that $\left(k^{(L)}\right)^{-1}(y)=\left(\widetilde{t}^{(L)}\right)^{-1}(y) \subset C C_{(L)}$ and that there is at least an element in $\left(\widetilde{t}^{(L)}\right)^{-1}(y)$, which is in $\mathcal{U}$. This means that $\mathcal{U} \cap C C_{(L)} \neq \varnothing$, which proves the density of $C C_{(L)}$.

Using the density of $C C_{(L)}$, the first frontier condition for the secondary stratification becomes obvious. For the second one, consider in $\mathcal{C}_{0}^{(L)}$ an arbitrary open neighborhood $\mathcal{U}$ of a point $x \in C S_{\left(H^{\prime}\right) \succ(L)}$. By the openness property of $k^{(L)}$, we obtain that $\mathcal{O}=k^{(L)}(\mathcal{U})$ is an open neighborhood of $k^{(L)}(x)$ in $\overline{Q^{(L)}}$. Applying (i), we have that $\mathcal{O} \cap Q^{(H)} \neq \varnothing \Longleftrightarrow\left(H^{\prime}\right) \succ(H) \succ(L)$. Furthermore, the surjectivity 
of $\widetilde{t}^{(H) \succ(L)}$ implies $\left(\widetilde{t}^{(H) \succ(L)}\right)^{-1}(z) \cap \mathcal{U} \neq \varnothing$ for any $z \in \mathcal{O} \cap Q^{(H)}$, proving that $C S_{\left(H^{\prime}\right) \succ(L)} \subset \partial C S_{(H) \succ(L)} \Longleftrightarrow\left(H^{\prime}\right) \succ(H) \succ(L)$.

As $k^{(L)}$ restricted to each piece of the secondary decomposition is surjective, Remark 5.1 immediately implies that this map is a stratified surjective submersion.

We will refer to the strata of the form $C S_{(H) \succ(L)}$ as contact seams due to their stitching role, which will be explained later in Remark 5.3

This theorem completes the topological description of each contact stratum $\mathcal{C}_{0}^{(L)}$ in terms of its secondary stratification. We shall now begin the investigation of geometrical aspects, namely to what extent the strata of this secondary stratification admit canonical contact structures in the sense that the 1-forms generating them are induced by some cosphere bundle structures compatible with the reduced contact form on the contact stratum. Thus, denote by

$$
\widetilde{\Psi}^{(H)}: C C_{(H)} \rightarrow\left(S^{*} Q^{(H)}, \Theta_{\Sigma}^{(H)}\right)
$$

the bundle isomorphism given by Theorem 4.1, where $\Theta_{\Sigma}^{(H)}$ is a contact form on the cosphere bundle of $Q^{(H)}$. Observe that the restricted projection onto the first factor

$$
\mathrm{p}_{1(H) \succ(L)}:\left(J_{c t(H)}^{-1}(0) \backslash\left\{0_{T^{*} Q_{(H)}}\right\}\right) \times\left(N^{*} Q_{(H)}\right)_{(L)} \rightarrow J_{c t(H)}^{-1}(0) \backslash\left\{0_{T^{*} Q_{(H)}}\right\}
$$

is $\mathbb{R}_{+}$and $G$-equivariant. So it descends to the surjective submersion

$$
\widetilde{\mathrm{p}}_{1}^{(H) \succ(L)}: C S_{(H) \succ(L)}^{\circ} \rightarrow C C_{(H)},
$$

where

$$
C S_{(H) \succ(L)}^{\circ}:=\frac{\pi_{+}\left(J_{c t(H)}^{-1}(0) \backslash\left\{0_{T^{*} Q_{(H)}}\right\} \times\left(N^{*} Q_{(H)}\right)_{(L)}\right)}{G}
$$

is an open and dense submanifold of the contact seam $C S_{(H) \succ(L)}$. Then, for any pair $(H) \succ(L)$, we have the following bundle map covering the identity on $Q^{(H)}$ :

$$
\widetilde{\Psi}^{(H) \succ(L)}:=\widetilde{\Psi}^{(H)} \circ \widetilde{\mathrm{p}}_{1}^{(H) \succ(L)}: C S_{(H) \succ(L)}^{\circ} \rightarrow S^{*} Q^{(H)},
$$

which is also a surjective submersion. We are now able to endow each cosphere-like stratum $C C_{(H)}$ and each $C S_{(H) \succ(L)}^{\circ}$ with 1-forms given by

$$
\left(C C_{(H)}, \eta_{(H)}:=\left(\widetilde{\Psi}^{(H)}\right)^{*} \Theta_{\Sigma}^{(H)}\right)
$$

and

$$
\left(C S_{(H) \succ(L)}^{\circ}, \eta_{(H) \succ(L)}:=\left(\widetilde{\Psi}^{(H) \succ(L)}\right)^{*} \Theta_{\Sigma}^{(H)}\right) .
$$

It is impossible to induce in this way a 1-form on the whole piece $C S_{(H) \succ(L)}$, and hence we are forced to restrict ourselves, for the time being, to $C S_{(H) \succ(L)}^{\circ}$. However, we will show later how to extend this form to the whole $C S_{(H) \succ(L)}$.

Theorem 2.1 gives the existence of an abstractly defined contact structure on each contact piece $C_{0}^{(L)}$ generated by a 1 -form $\theta_{\sigma 0}^{(L)}$. One of the aims of this section is to investigate the compatibility of the previously defined forms $\eta_{(H)}$ and $\eta_{(H) \succ(L)}$ with the reduced contact form $\theta_{\sigma_{0}}{ }^{(L)}$ and to describe as much as possible this abstract contact structure. 


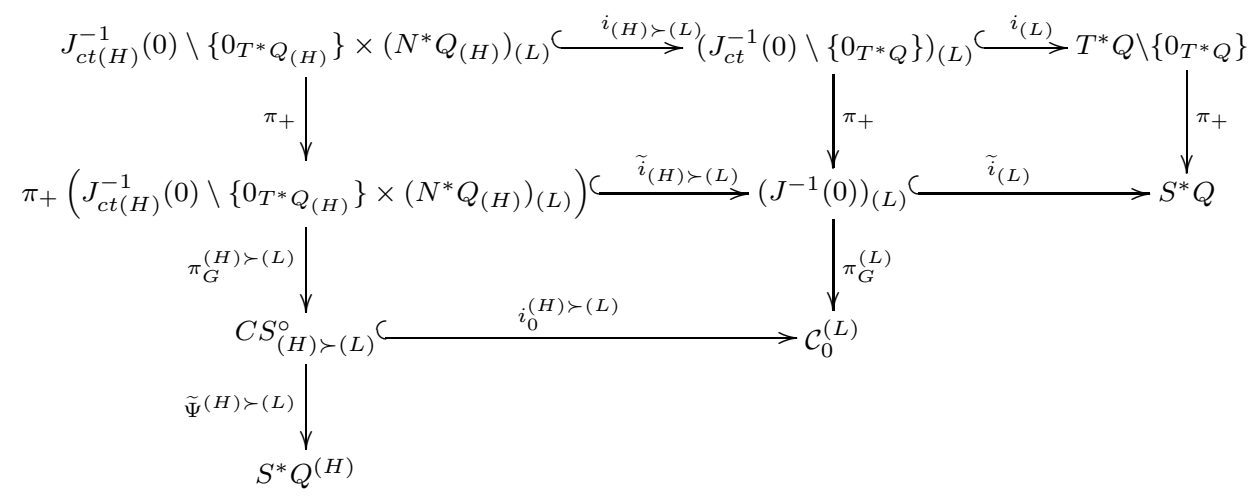

FIGURE 1. Diagram defining $\eta_{(H)}$

Theorem 5.2. The strata $C C_{(L)}$ and $C S_{(H) \succ(L)}^{\circ}$ within the contact stratum $\mathcal{C}_{0}^{(L)}$ satisfy the following properties:

(i) $\left(C C_{(L)}, \eta_{(L)}\right)$ is an open dense contact submanifold of the contact stratum $\mathcal{C}_{0}^{(L)}$ contactomorphic to $\left(S^{*}\left(Q^{(L)}\right), \Theta_{\Sigma}^{(L)}\right)$.

(ii) Using the above notation, the conformal classes of $\eta_{(L)}$ and $\eta_{(H) \succ(L)}$ admit smooth extensions to $\mathcal{C}_{0}^{(L)}$ equivalent to $\theta_{\sigma_{0}}^{(L)}$, namely

$$
\left.\theta_{\sigma 0}^{(L)}\right|_{C C_{(L)}} \simeq \eta_{(L)} \quad \text { and }\left.\quad \theta_{\sigma 0}^{(L)}\right|_{C S_{(H) \succ(L)}^{\circ}} \simeq \eta_{(H) \succ(L)} .
$$

The extension of $\eta_{(L)}$ is unique.

(iii) The conformal class of $\eta_{(H) \succ(L)}$ can be smoothly and uniquely extended to the whole stratum $C S_{(H) \succ(L)}$. If $(H) \in I_{Q}^{*}$, then $C S_{(H) \succ(L)}$ is a coisotropic submanifold of the contact stratum $\mathcal{C}_{0}^{(L)}$. When $(H) \in I_{Q} \backslash I_{Q}^{*}$, then $C S_{(H) \succ(L)}$ is a Legendrian submanifold of the contact stratum $\mathcal{C}_{0}^{(L)}$.

Proof. (i) is a simple consequence of Theorem 4.1

For (ii), let $(L)$ and $(H)$ be two fixed elements of $I_{Q}^{*}$ and $I_{Q}$ respectively and $i_{0}^{(H) \succ(L)}: C S_{(H) \succ(L)}^{\circ} \rightarrow \mathcal{C}_{0}^{(L)}$ the inclusion map. By definition,

$$
\begin{aligned}
& \left.\theta_{\sigma 0}^{(L)}\right|_{C S_{(H)}^{\circ} \succ(L)} \simeq \eta_{(H) \succ(L)} \Longleftrightarrow \exists f>0 \quad \text { in } \quad C^{\infty}\left(C S_{(H) \succ(L)}\right) \quad \text { such that } \\
& \left.\theta_{\sigma 0}{ }^{(L)}\right|_{C S_{(H) \succ(L)}^{\circ}}=f \eta_{(H) \succ(L)} \quad \Longleftrightarrow \quad\left(i_{\circ}^{(H) \succ(L)}\right)^{*} \theta_{\sigma 0}^{(L)} \simeq\left(\widetilde{\Psi}^{(H) \succ(L)}\right)^{*} \Theta_{\Sigma}^{(H)} .
\end{aligned}
$$

To simplify the reading of the proof, consider Figures 11 and 2, where $\pi_{G}^{(H) \succ(L)}$ and $\bar{\pi}_{G}^{(H)}$ denote the canonical $G$-projections and all the horizontal arrows in the first and second diagram are injections and projections respectively.

As $\pi_{G}^{(H) \succ(L)} \circ \pi_{+}$is a submersion, it suffices to prove that

$$
\left(i_{0}^{(H) \succ(L)} \circ \pi_{G}^{(H) \succ(L)} \circ \pi_{+}\right)^{*} \theta_{\sigma 0}^{(L)} \simeq\left(\widetilde{\Psi}^{(H) \succ(L)} \circ \pi_{G}^{(H) \succ(L)} \circ \pi_{+}\right)^{*} \Theta_{\Sigma}^{(H)} .
$$




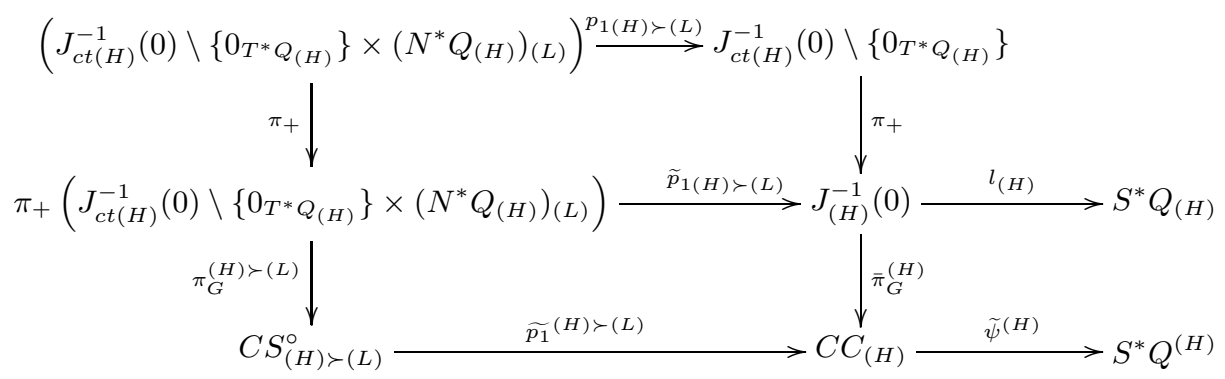

FiguRE 2. Diagram defining $\eta_{(H) \succ(L)}$

Observe that $i_{0}^{(H) \succ(L)} \circ \pi_{G}^{(H) \succ(L)} \circ \pi_{+}=\pi_{G}^{(L)} \circ \pi_{+} \circ i_{(H) \succ(L)}$ and the first term of (5.4) becomes

$$
\begin{array}{r}
\left(\pi_{G}^{(L)} \circ \pi_{+} \circ i_{(H) \succ(L)}\right)^{*} \theta_{\sigma 0}^{(L)}=i_{(H) \succ(L)}^{*} \circ \pi_{+}^{*}\left(\left(\pi_{G}^{(L)}\right)^{*} \theta_{\sigma 0}^{(L)}\right) \\
=i_{(H) \succ(L)}^{*} \circ \pi_{+}^{*}\left(\widetilde{i}_{(L)}^{*} \theta_{\sigma}\right)=\left(\pi_{+} \circ j_{(H)} \circ \Phi\right)^{*} \theta_{\sigma},
\end{array}
$$

where in the last line we have used Theorem 2.1 together with the equality $\pi_{+} \circ$ $j_{(H)} \circ \Phi=\widetilde{i}_{(L)} \circ \pi_{+} \circ i_{(H) \succ(L)}$, with $j_{(H)}$ and $\Phi$ inclusions defined by

$$
\Phi:\left.\left(J_{c t(H)}^{-1}(0) \backslash\left\{0_{T^{*} Q_{(H)}}\right\} \times\left(N^{*} Q_{(H)}\right)_{(L)}\right) \hookrightarrow T^{*} Q\right|_{Q_{(H)}} \backslash\left\{0_{T^{*} Q_{(H)}}\right\}
$$

and

$$
j_{(H)}:\left.T^{*} Q\right|_{Q_{(H)}} \backslash\left\{0_{T^{*} Q_{(H)}}\right\} \hookrightarrow T^{*} Q \backslash\left\{0_{T^{*} Q}\right\} .
$$

Using this time $\bar{\pi}_{G}^{(H)} \circ \pi_{+} \circ p_{1(H) \succ(L)}=\widetilde{p}_{1}^{(H) \succ(L)} \circ \pi_{G}^{(H) \succ(L)} \circ \pi_{+}$, we can write the second term of (5.4) as

$$
\begin{gathered}
\left(\widetilde{\Psi}^{(H)} \circ \widetilde{p}_{1}^{(H) \succ(L)} \circ \pi_{G}^{(H) \succ(L)} \circ \pi_{+}\right)^{*} \Theta_{\Sigma}^{(H)}=\left(\widetilde{\Psi}^{(H)} \circ \bar{\pi}_{G}^{(H)} \circ \pi_{+} \circ p_{1(H) \succ(L)}\right)^{*} \Theta_{\Sigma}^{(H)} \\
\quad=\left(\pi_{+} \circ p_{1(H) \succ(L)}\right)^{*}\left(\widetilde{\Psi}^{(H)} \circ \bar{\pi}_{G}^{(H)}\right)^{*} \Theta_{\Sigma}^{(H)} \simeq\left(\pi_{+} \circ p_{1(H) \succ(L)}\right)^{*} l_{(H)}^{*} \theta_{(H) \Sigma},
\end{gathered}
$$

where $\theta_{(H) \Sigma}$ is a contact form on $S^{*} Q_{(H)}$. Let $p_{(H)}:\left.T^{*} Q\right|_{Q_{(H)}} \backslash\left\{0_{T^{*} Q_{(H)}}\right\} \rightarrow$ $T^{*} Q_{(H)}$ be the projection map. Since $l_{(H)} \circ \pi_{+} \circ p_{1(H) \succ(L)}=\pi_{+} \circ p_{(H)} \circ \Phi$, the second term is in the same conformal class as $\Phi^{*} p_{(H)}^{*} \pi_{+}^{*} \theta_{(H) \Sigma}$ and, hence, equation (5.4) is equivalent to

$$
\begin{aligned}
\Phi^{*} p_{(H)}^{*} \pi_{+}^{*} \theta_{(H) \Sigma} \simeq \Phi^{*} j_{(H)}^{*} \pi_{+}^{*} \theta_{\sigma} & \Longleftrightarrow \Phi^{*} p_{(H)}^{*}\left(\Sigma \circ \pi_{+}\right)^{*} \theta_{(H)} \simeq \Phi^{*} j_{(H)}^{*}\left(\sigma \circ \pi_{+}\right)^{*} \theta \\
& \Longleftrightarrow \Phi^{*} p_{(H)}^{*} f_{\Sigma} \theta_{(H)} \simeq \Phi^{*} j_{(H)}^{*} f_{\sigma} \theta,
\end{aligned}
$$

where $\theta$ and $\theta_{(H)}$ are the canonical one-forms on $T^{*} Q$ and $T^{*} Q_{(H)}$ respectively, and $\sigma, \Sigma$ are sections in the associated cosphere bundles. But $p_{(H)}^{*} \theta_{(H)}=j_{(H)}^{*} \theta$, as can easily be seen in local coordinates, which proves (5.4).

As for the extension of the conformal class of $\eta_{(L)}$, an analogous proof can be developed just by considering the limit case $(H)=(L)$, when $C S_{(H) \succ(L)}^{0}$ degenerates in $C C_{(L)}$. In order to prove the uniqueness of this extension, let us consider a point $x \in \mathcal{C}_{0}^{(L)}$ and one tangent vector $v_{x} \in T_{x} \mathcal{C}_{0}^{(L)}$. As $C C_{(L)}$ is open and dense in $\mathcal{C}_{0}^{(L)}$, there is a sequence of points $x_{k} \in C C_{(L)}$ and one of vectors 
$v_{x_{k}} \in T_{x_{k}} C C_{(L)} \simeq T_{x_{k}} \mathcal{C}_{0}^{(L)}$ such that

$$
\lim _{k \rightarrow \infty} x_{k}=x, \quad \lim _{k \rightarrow \infty} v_{x_{k}}=v_{x} .
$$

From the above arguments and using the continuity of $\theta_{\sigma 0}^{(L)}$, we have that

$$
\lim _{k \rightarrow \infty} \frac{\eta_{(L)}\left(x_{k}\right)\left(v_{x_{k}}\right)}{g\left(x_{k}\right)}=\lim _{k \rightarrow \infty} \theta_{\sigma 0}{ }^{(L)}\left(x_{k}\right)\left(v_{x_{k}}\right)=\theta_{\sigma 0}{ }^{(L)}(x)\left(v_{x}\right),
$$

with $g \in C^{\infty}\left(C C_{(L)}\right)$ a positive function such that $\eta_{(L)}=\left.g \theta_{\sigma 0}{ }_{\sigma}^{(L)}\right|_{C C_{(L)}}$. We have thus proved that the class of $\theta_{\sigma_{0}}^{(L)}$ is the unique smooth extension of the class of $\eta_{(L)}$ to $\mathcal{C}_{0}^{(L)}$.

(iii) To extend the class of $\eta_{(H) \succ(L)}$ from $C S_{(H) \succ(L)}^{\circ}$ to the whole piece $C S_{(H) \succ(L)}$, we will apply the same type of arguments as before, using this time that $C S_{(H) \succ(L)}^{\circ}$ is open and dense in $C S_{(H) \succ(L)}$. Namely, for any point $x \in C S_{(H) \succ(L)}$ and any $v_{x} \in T_{x} C S_{(H) \succ(L)}$, there is a sequence of points $x_{k} \in C S_{(H) \succ(L)}^{\circ}$ and one of vectors $v_{x_{k}} \in T_{x_{k}} C S_{(H) \succ(L)}^{\circ} \simeq T_{x_{k}} C S_{(H) \succ(L)}$ such that

$$
\lim _{k \rightarrow \infty} x_{k}=x, \quad \lim _{k \rightarrow \infty} v_{x_{k}}=v_{x} .
$$

Observe that

$$
\lim _{k \rightarrow \infty} \frac{\eta_{(H) \succ(L)}\left(x_{k}\right)\left(v_{x_{k}}\right)}{f\left(x_{k}\right)}=\theta_{\sigma 0}^{(L)}(x)\left(v_{x}\right),
$$

and notice that this extension is also unique and given by the conformal class of $\left.\theta_{\sigma 0}{ }^{(L)}\right|_{C S_{(H) \succ(L)}}$.

To check the coisotropy and Legendrian submanifold conditions, let $x \in$ $C S_{(H) \succ(L)}^{\circ}$. A direct count of dimensions gives

$$
\operatorname{dim} \operatorname{ker} \theta_{\sigma 0}^{(L)}(x)=\operatorname{dim} \mathcal{C}_{0}^{(L)}-1=2\left(\operatorname{dim} Q_{(L)}-\operatorname{dim} G+\operatorname{dim} L-1\right)
$$

since $S^{*} Q^{(L)}$ is open in the corresponding contact stratum. At this point we need the following intermediate result.

Lemma 5.1. The dimension of the tangent space to a contact seam is

$$
\operatorname{dim} T_{x} C S_{(H) \succ(L)}=\operatorname{dim} Q_{(H)}+\operatorname{dim} Q_{(L)}-2 \operatorname{dim} G+\operatorname{dim} H+\operatorname{dim} L-1 .
$$

Proof. We want to compute $\operatorname{dim} T_{x} C S_{(H) \succ(L)}=\operatorname{dim} C S_{(H) \succ(L)}$. For this, let $\pi(z)=k^{0}(x)$ be the base point of $x$, where $z \in Q_{(H)}$ with $G_{z}=H$ and note that $\operatorname{dim} C S_{(H) \succ(L)}=\operatorname{dim}\left(J_{c t}^{-1}(0) \cap T_{z}^{*} Q\right)_{(L)}+\operatorname{dim} Q_{(H)}-\operatorname{dim} G+\operatorname{dim} L-1$, where the class $(L)$ refers to the linear $H$-action on the vector space $J_{c t}^{-1}(0) \cap T_{z}^{*} Q$. On the other hand, the inverse of the Riemannian bundle isomorphism $T Q \rightarrow T^{*} Q$ maps $\left(J_{c t}^{-1}(0) \cap T_{z}^{*} Q\right)_{(L)} H$-equivariantly isomorphically to $\left(S_{z}\right)_{(L)}$. Now, if $\psi$, $U$, and $U^{\prime}$ are as in the Tube Theorem (2.2), then $\psi$ restricts to a diffeomorphism between $G \times_{H}\left(\left(S_{z}\right)_{(L)} \cap U\right)$ and $U \cap Q_{(L)}$. Since $\operatorname{dim} G \times_{H}\left(S_{z}\right)_{(L)}=$ $\operatorname{dim} G+\operatorname{dim}\left(S_{z}\right)_{(L)}-\operatorname{dim} H$, we can compute

$$
\operatorname{dim}\left(S_{z}\right)_{(L)}=\operatorname{dim} Q_{(L)}-\operatorname{dim} G+\operatorname{dim} H .
$$

Finally we obtain $\operatorname{dim} T_{x} C S_{(H) \succ(L)}=\operatorname{dim} Q_{(H)}+\operatorname{dim} Q_{(L)}-2 \operatorname{dim} G+\operatorname{dim} H+$ $\operatorname{dim} L-1$. 
Consequently, a simple dimension count gives

$$
\begin{aligned}
\operatorname{dim} T_{x} C S_{(H) \succ(L)}-\frac{1}{2} \operatorname{dim} \operatorname{ker} \theta_{\sigma 0}^{(L)}(x) & =\operatorname{dim} Q_{(H)}-\operatorname{dim} G+\operatorname{dim} H \\
& =\operatorname{dim}\left(S_{z}\right)_{(H)} \geq 0,
\end{aligned}
$$

where $z \in Q_{(H)}$ is the base point of $x$ and $S_{z}$ is the associated linear slice. Suppose first that $(H) \in I_{Q}^{*}$ and so $\operatorname{dim} T_{x} C S_{(H) \succ(L)}-\frac{1}{2} \operatorname{dim} \operatorname{ker} \theta_{\sigma 0}{ }^{(L)}(x) \supsetneqq 0$. This implies that $C S_{(H) \succ(L)}^{\circ}$ and $C S_{(H) \succ(L)}$ can be neither isotropic nor Legendrian submanifolds of $\mathcal{C}_{0}^{(L)}$ and that $T_{x} C S_{(H) \succ(L)}^{\circ} \nsubseteq \operatorname{ker} \theta_{\sigma 0}{ }^{(L)}(x)$ for any $x \in C S_{(H) \succ(L)}^{\circ}$.

Now let

$$
W_{x}:=T_{x} C S_{(H) \succ(L)}^{\circ} \cap \operatorname{ker} \theta_{\sigma 0}^{(L)}(x)=T_{x} C S_{(H) \succ(L)} \cap \operatorname{ker} \theta_{\sigma 0}{ }^{(L)}(x)
$$

and

$$
V_{x}:=\left\{v \in T_{x} C S_{(H) \succ(L)}^{\circ} \backslash \operatorname{ker} \theta_{\sigma_{0}}^{(L)}(x): v=v_{0} \oplus k R(x), k \in \mathbb{R}, v_{0} \in \operatorname{ker} \theta_{\sigma 0}{ }^{(L)}(x)\right\} .
$$

One can easily check that $V_{x}$ is a one-dimensional vector space and that for any $x \in$ $C S_{(H) \succ(L)}^{\circ}$, we have $T_{x} C S_{(H) \succ(L)}^{\circ}=W_{x} \oplus V_{x}$. As $\widetilde{\Psi}^{(H) \succ(L)}$ is a surjective submersion and $\left.\theta_{\sigma 0}^{(L)}\right|_{C S_{(H) \succ(L)}^{\circ}} \simeq \eta_{(H) \succ(L)}$, it follows that $T_{x} \widetilde{\Psi}^{(H) \succ(L)}\left(W_{x}\right)=\operatorname{ker} \Theta_{\Sigma}^{(H)}(y)$ and $T_{x} \widetilde{\Psi}^{(H) \succ(L)}\left(V_{x}\right)=\operatorname{span}\left\{R_{\Sigma}(y)\right\}$, where $y=\widetilde{\Psi}^{(H) \succ(L)}(x)$ and $R_{\Sigma}(y)$ is the Reeb vector field of $\left(S^{*} Q^{(H)}, \Theta_{\Sigma}^{(H)}\right)$. Therefore, we obtain

$$
\begin{aligned}
& \left.\operatorname{rank} d \eta_{(H) \succ(L)}(x)\right|_{W_{x}}=\operatorname{dim} W_{x}-\left.\operatorname{dim} \operatorname{ker} d \eta_{(H) \succ(L)}(x)\right|_{W_{x}} \\
& \quad=\operatorname{dim} W_{x}-\operatorname{dim}\left\{v \in W_{x}: d \Theta_{\Sigma}^{(H)}(y)\left(T_{x} \widetilde{\Psi}^{(H) \succ(L)} v, T_{x} \widetilde{\Psi}^{(H) \succ(L)} w\right)=0, \forall w \in W_{x}\right\} \\
& \quad=\operatorname{dim} W_{x}-\left.\operatorname{dim} \operatorname{ker} T_{x} \widetilde{\Psi}^{(H) \succ(L)}\right|_{W_{x}}=\operatorname{dim} S^{*} Q^{(H)}-1 .
\end{aligned}
$$

This shows that $\left.\operatorname{rank} d \eta_{(H) \succ(L)}(x)\right|_{W}=2 \operatorname{dim} W-\left(\operatorname{dim} \mathcal{C}_{0}^{(L)}-1\right)$ proving that $C S_{(H) \succ(L)}^{\circ}$ is a coisotropic submanifold. Since $C S_{(H) \succ(L)}^{\circ}$ is dense in $C S_{(H) \succ(L)}$, by an extension argument similar to the one used before, we have that $C S_{(H) \succ(L)}$ is also a coisotropic submanifold of the corresponding contact stratum.

If $(H) \in I_{Q} \backslash I_{Q}^{*}$, then $\operatorname{dim} T_{x} C S_{(H) \succ(L)}=\frac{1}{2} \operatorname{dim} \operatorname{ker} \theta_{\sigma 0}^{(L)}(x)$ and by the definition (5.3), $\eta_{(H) \succ(L)}=0$ since $S^{*} Q^{(H)}$ is the trivial bundle, proving thus that $C S_{(H) \succ(L)}$ is a Legendrian submanifold of $\mathcal{C}_{0}^{(L)}$.

Remark 5.2. Note that the contact seams $C S_{(H) \succ(L)}$ can never be contact submanifolds of $\mathcal{C}_{0}^{(L)}$.

5.2. The C-L stratification of $\mathcal{C}_{0}$. In this subsection we prove the existence of a new stratification of the contact reduced space $\mathcal{C}_{0}$, different from the contact stratification in Theorem 2.1. The existence of this new stratification, which we call the C-L stratification since its strata are coisotropic or Legendrian submanifolds of the corresponding contact stratum, is due to the bundle structure of the contact manifold that we start with. We will see that the C-L stratification is strictly finer than the contact one if the base manifold $Q$ has more than one orbit type. In principle, this is not an advantage since the contact stratification partitions the singular contact quotient in fewer and larger smooth components. However, if we take into account the bundle structure of the problem, we can see why this new stratification is more appropriate. 
The most important feature of regular cosphere bundle reduction, Theorem 3.1 . is that if we start with the cosphere bundle of a manifold $Q$, we end up again with a cosphere bundle, this time over $Q / G$. Furthermore, the reduced contact structure on $S^{*}(Q / G)$ equals the canonical cosphere contact structure. In the singular setting, however, the lack of smoothness of the quotient spaces involved forces us to choose another definition of fibration. The most natural one when working with decomposed or stratified spaces is the following: if $A$ and $B$ are decomposed spaces together with a continuous surjection $f: A \rightarrow B$, we say that $f: A \rightarrow B$ defines a stratified bundle over $B$ if $f$ is a morphism of decomposed spaces. In our case, there is a natural projection $k^{0}: \mathcal{C}_{0} \rightarrow Q / G$ induced from the cosphere bundle projection $k: S^{*} Q \rightarrow Q$. If we consider the natural orbit type stratification of $Q / G$ and the contact one of $\mathcal{C}_{0}$, then the projection does not define a stratified bundle over $Q / G$ since the image of a contact stratum $\mathcal{C}_{0}^{(L)}$ under the projection is $\overline{Q^{(L)}}$, which includes several orbit type strata of $Q / G$. We will prove that the choice of the coisotropic stratification for the contact quotient $\mathcal{C}_{0}$ solves this problem.

Consider the partition of $\mathcal{C}_{0}$ obtained by putting together all the secondary strata found in every contact stratum:

$$
\mathcal{C}_{0}=\coprod_{(L)} C C_{(L)} \coprod_{\left(K^{\prime}\right) \succ(K)} C S_{\left(K^{\prime}\right) \succ(K)}
$$

for every pair of classes $(L),(K) \in I_{Q}^{*}$ and every $\left(K^{\prime}\right) \in I_{Q}$.

Theorem 5.3. The partition $(5.6)$ is a decomposition of $\mathcal{C}_{0}$ inducing a stratification, called the $C$-L stratification, that satisfies the following properties:

(1) If $Q / G$ is connected and $\left(L_{0}\right)$ is the principal orbit type in $Q$, then $C C_{\left(L_{0}\right)}$ is open and dense in $\mathcal{C}_{0}$.

(2) $k^{0}: \mathcal{C}_{0} \rightarrow Q / G$ is a stratified bundle with respect to the $C$ - $L$ stratification of $\mathcal{C}_{0}$ and the orbit type stratification of $Q / G$.

(3) If $I_{Q}$ consists of more than one class, the $C$ - $L$ stratification is strictly finer than the contact one, and they are identical otherwise.

(4) The frontier conditions for the $C$ - $L$ stratification of $\mathcal{C}_{0}$ are:

(i) $C C_{(K)} \subset \partial C C_{(H)} \Longleftrightarrow(H) \prec(K)$,

(ii) $C S_{(K) \succ(H)} \subset \partial C C_{(H)} \Longleftrightarrow(H) \prec(K)$,

(iii) $C_{(K)} \subset \partial C S_{(K) \succ(H)} \Longleftrightarrow(H) \prec(K)$,

(iv) $C S_{\left(K^{\prime}\right) \succ(H)} \subset \partial C S_{(K) \succ(H)} \Longleftrightarrow(H) \prec(K) \prec\left(K^{\prime}\right)$,

(v) $C S_{(K) \succ\left(H^{\prime}\right)} \subset \partial C S_{(K) \succ(H)} \Longleftrightarrow(H) \prec\left(H^{\prime}\right) \prec(K)$.

Proof. For (1), recall by Proposition 4.1 that $I_{J^{-1}(0)}=I_{Q}^{*}$. The principal orbit type of the isotropy lattice corresponds to an open and dense piece, so $\left(J^{-1}(0)\right)_{\left(L_{0}\right)}$ is open and dense in $J^{-1}(0)$, since $\left(L_{0}\right)$ is by hypothesis the principal orbit type in $I_{Q}^{*}$ (assuming that $\operatorname{dim} Q \neq 0$ ) and hence in $I_{J^{-1}(0)}$. Consequently, as the orbit map $J^{-1}(0) \rightarrow \mathcal{C}_{0}$ is continuous and open, $\mathcal{C}_{0}^{\left(L_{0}\right)}$ is open and dense in $\mathcal{C}_{0}$. Now, since $\mathcal{C}_{0}^{\left(L_{0}\right)}$ is equipped with the relative topology with respect to $\mathcal{C}_{0}$ and $C C_{\left(L_{0}\right)}$ is open and dense in it (Theorem 5.1), it follows that $C C_{\left(L_{0}\right)}$ is also open and dense in $\mathcal{C}_{0}$. For (2), note that the restrictions of $k^{0}$ to $C C_{(L)}$ and $C S_{(H) \succ(L)}$ coincide with the corresponding restrictions of $k^{(L)}$, which, by Remark 5.1, are smooth surjective submersions over $Q^{(L)}$ and $Q^{(H)}$ respectively for every $(L) \in I_{Q}^{*}$ and $(H) \in I_{Q}$. This 
shows that these restrictions map each C-L stratum of $\mathcal{C}_{0}$ to an orbit type stratum of $Q / G$. Therefore, $k^{0}$ is a morphism of stratified spaces. To prove (3), recall from Theorem 4.1 that if $I_{Q}$ consists of a single orbit type $(H)$, then $\mathcal{C}_{0}=\mathcal{C}_{0}^{(H)}=C C_{(H)}$ (assuming $\operatorname{dim} Q \neq 0$ ) and its contact and C-L stratifications are both trivial and identical. If there is more than one orbit type in the base, the number of C-L strata is strictly greater than the number of contact strata (which is equal to the number of orbit types of $I_{Q}^{*}$ ). The identity map in $\mathcal{C}_{0}$ injects each C-L stratum in the unique contact stratum to which it belongs and is hence a morphism of stratified spaces. Therefore, the C-L stratification is finer than the contact one. For (4), relations (ii) and $(i v)$ follow from the frontier conditions of the secondary stratum $\mathcal{C}_{0}^{(H)}$. To prove $(i)$, it suffices to recall from the general theory of singular contact reduction that $\mathcal{C}_{0}^{(K)} \subset \partial \mathcal{C}_{0}^{(H)}$ if and only if $(H) \prec(K)$. Using the density of any maximal secondary stratum $C C_{(L)}$ in the corresponding contact piece $\mathcal{C}_{0}^{(L)},(i)$ follows. (iii) is a consequence of $(v)$ if one considers the limit case $C C_{(K)}=C S_{(K) \succ(K)}$.

Finally, to prove $(v)$, choose a point $[x] \in C S_{(K) \succ\left(H^{\prime}\right)} \subset \mathcal{C}_{0}$ and an open neighborhood $[x] \in O \subset \mathcal{C}_{0}$. We shall show that $O \cap C S_{(K) \succ(H)} \neq \varnothing$ if $(H) \prec\left(H^{\prime}\right) \prec(K)$. Let $x \in J^{-1}(0)$ be a preimage of $[x]$. We can assume without loss of generality that $G_{x}=H^{\prime}$ and that the projection of $x$, i.e. the point $z=k(x) \in Q$, satisfies $G_{z}=K$. Let $U$ be the only open $G$-saturated set in $J^{-1}(0)$ such that $U / G=O$. Then, identifying $S^{*} Q$ with the unit bundle in $T^{*} Q$ via a $G$-invariant metric on $Q$, we have that $x$ is a unit covector lying in the subset of the cotangent fiber at $z$ given by $\left(S_{z}^{K}\right)^{*} \oplus\left(N_{z}^{*} Q_{(K)}\right)_{\left(H^{\prime}\right)}$. By the general properties of linear representations of compact groups on vector spaces and the property (iii) of cotangent-lifted actions in Lemma 4.2. it follows that $p_{2}\left(U \cap T_{z}^{*} Q\right) \cap\left(N_{z}^{*} Q_{(K)}\right)_{(H)} \neq \varnothing$ for every compact subgroup $H$ of $K$ such that $H \prec H^{\prime}$ and $\left(N_{z}^{*} Q_{(K)}\right)_{(H)} \neq \varnothing$, i.e., $(H) \in I_{Q}$. Here, $p_{2}$ is the linear projection $\left(S_{z}^{K}\right)^{*} \oplus N_{z}^{*} Q_{(K)} \rightarrow N_{z}^{*} Q_{(K)}$. From this, it follows that if $x^{\prime} \in p_{2}\left(U \cap T_{z}^{*} Q\right) \cap\left(N_{z}^{*} Q_{(K)}\right)_{(H)}$, then $\left[x^{\prime}\right] \in O \cap C S_{(K) \succ(H)}$.

Remark 5.3. The previous result shows that, identifying a stratum $C C_{(H)}$ with $S^{*} Q_{(H)}$ as shown in Theorem [5.2, the reduced space $\mathcal{C}_{0}$ is almost everywhere a collection of cosphere bundles, one for each orbit type stratum of positive dimension in $Q / G$. These cosphere bundles satisfy the same frontier conditions as their bases, i.e., $S^{*} Q^{(K)} \subset \partial S^{*} Q^{(H)}$ if and only if $Q^{(K)} \subset \partial Q^{(H)}$ (condition (i)), but in this case there is always a contact seam $C S_{(K) \succ(H)}$ between them, which "glues together" these two cosphere bundles, as reflected in conditions (ii) and (iii).

5.3. A remark on the local properties of the C-L stratification of $\mathcal{C}_{0}$. Throughout this paper we have used a purely topological concept of stratification (see subsection 2.1). However, in the literature most of the time the notion of stratification is a finer one, in a sense incorporating some sort of smooth structure not confined to each stratum. Namely, the additional condition usually imposed on a stratified space $X$ is that of being a locally trivial cone space (which together with a smooth structure of degree $\geq 2$ implies that $X$ is a Whitney space, see [18] for details).

According to [12, the contact quotient $\mathcal{C}_{0}$ together with the stratification given by Theorem 2.1 is a locally trivial cone space. In that paper, the authors prove this fact using a contact analogue of the equivariant symplectic tubular neighborhood of Marle, Guillemin and Sternberg. They study the conical properties of the stratification in the local model provided by the corresponding equivariant tube $\phi: \mathcal{C} \rightarrow U$. 
This is possible since the basic ingredients to construct the strata, the orbit types $\mathcal{C}_{(H)}$, are mapped in the local model to $U_{(H)}$. However, this is not the case for the cosphere bundle. The building blocks of the secondary and C-L stratifications of a cosphere bundle quotient are $\pi_{+}\left(s_{(L)} \backslash\left\{0_{T^{*} Q_{(L)}}\right\}\right)$ and $\pi_{+}\left(s_{(H) \succ(L)}\right)$. In order to express them in the tubular neighborhood, one would need the tube $\phi$ to be explicitly defined or at least adapted to the cosphere bundle category in a way that reflects the fibrated nature of $\mathcal{C}$. Consequently, the problem of studying the local triviality of the secondary or C-L stratifications implies finding such an adapted normal form for cosphere bundles, which is yet unknown.

\section{Singular aCtions on the Base With REgular LifTS TO THE COSPHERE BUNDLE}

In the following definition we introduce a class of actions which may have singularities on $Q$ but that will be proven to yield regular lifted actions on $S^{*} Q$.

Definition 6.1. An almost semifree action of $G$ on $Q$ is a smooth action such that a) it is free almost everywhere, b) the connected components of every orbit of non-maximal dimension are isolated, and c) for every non-trivial isotropy subgroup $H \in I_{Q}$ with Lie algebra $\mathfrak{h}$, its induced adjoint representation on $(\mathfrak{g} / \mathfrak{h}) \backslash\{0\}$ given by $h \cdot[\xi]=\left[\operatorname{Ad}_{h} \xi\right]$ is free.

Note that for any almost semifree action, the quotient space $Q / G$ consists of an open and dense stratum $Q^{(e)}$, except possibly for a set of isolated singular points. The next proposition shows that the class of almost semifree actions is in one-to-one correspondence with the class of free actions on $S^{*} Q$.

Proposition 6.1. Let $S^{*} Q$ be the cosphere bundle of $Q$ endowed with the lift of a proper action of a Lie group $G$ on $Q$. This lifted action is free if and only if the action on $Q$ is almost semifree.

Proof. Recall that, identifying, with the help of a $G$-invariant Riemannian metric, $S^{*} Q$ with the unit bundle $S Q \subset T Q$ and $T Q$ with $T^{*} Q, G$ acts freely on $S^{*} Q$ if and only if its tangent-lifted action on $T Q$ is free on the unit bundle, and hence if it is free away from the zero section (since by linearity the lifted action intertwines the fiber, rescaling by non-zero factors). Let $q \in Q$ with stabilizer $G_{q}=H \neq\{e\}$, $S \subset T_{q} Q$ a linear slice for the $G$-action at $q$ and $v=\xi_{Q}(q)+s \in T_{q} Q \backslash\{0\}$. Note that all the admissible $\xi$ 's differ by an element of the Lie algebra of $H$. Then $U=G \cdot \exp _{q}(S)$ is a $G$-invariant neighborhood of the orbit $G \cdot q=G \cdot \exp _{q}(0)$ and there is an $H$-isomorphism $f: T_{q} Q \rightarrow \mathfrak{g} / \mathfrak{h} \times S$ given by $f\left(\xi_{Q}(q)+s\right)=([\xi], s)$, where the $H$-invariance is with respect to the linear action on $T_{q} Q$ and the diagonal action on $\mathfrak{g} / \mathfrak{h} \times S$ given by $h \cdot([\xi], s)=\left(\left[\operatorname{Ad}_{h} \xi\right], h \cdot s\right)$. Consequently, $G_{v}=H_{v}=H_{s} \cap H_{[\xi]}$.

Suppose first that the lifted action of $G$ on $S^{*} Q$ is free. Then any point $q^{\prime} \in$ $U \backslash G \cdot q$ can be written as $q^{\prime}=g \cdot \exp _{q}(s)$ for some $0 \neq s \in S$ with $g \in G$ and $G_{q^{\prime}}=g H_{s} g^{-1}=\{e\}$, since $G_{s}=H_{s}=\{e\}$ as assumed above. Hence the $G$-action on $Q$ is almost semifree.

For $v=\xi_{Q}\left(q_{0}\right) \in T_{q_{0}} Q \backslash\{0\}$ with $\xi \in \mathfrak{g}, \xi \notin \mathfrak{h}$ we obtain that $G_{v}=\{e\}=H_{v}=$ $H_{[\xi]}$, thus proving that the induced adjoint representation on $(\mathfrak{g} / \mathfrak{h}) \backslash\{0\}$ is free.

To prove the converse implication, let $v \in T_{q} Q \backslash\{0\}$ as before, with $v=s+\xi_{Q}\left(q_{0}\right)$, where $s \in S$ and $\xi \in \mathfrak{g}$. If $s$ is different from zero, multiplying it if necessary by a positive scalar smaller than one, we can guarantee that $G \cdot \exp _{q}(s) \subset U \backslash G \cdot q$. 
Shrinking $U$ if necessary, we can guarantee that all of the points in $U \backslash G \cdot q_{0}$ have trivial isotropy, since the orbits of non-maximal dimension are isolated by hypothesis. Using again the Tube Theorem, the isotropy groups of these points are $g H_{s} g^{-1}=\{e\}$, for every $g \in G$, which forces $H_{s}=\{e\}$ and hence $H_{v}=\{e\}$. In the case when $s=0$, we have that $G_{v}=H_{[\xi]}=\{e\}$, thus completing the proof.

Remark 6.1. To geometrically express the third condition in Definition 6.1 notice that every non-trivial isotropy subgroup $H=G_{q} \in I_{Q}$ acts freely on $(\mathfrak{g} / \mathfrak{h}) \backslash\{0\}$ if and only if for any element $h \in H$ the associated diffeomorphism of $Q$ maps bijectively $\left\{\exp _{q}(t \xi) \cdot q: t \in \mathbb{R}\right\}$ to $\left\{\exp _{q}\left(t \operatorname{Ad}_{h} \xi\right) \cdot q: t \in \mathbb{R}\right\}$ for every $\xi \in \mathfrak{g}$ with $[\xi] \neq 0$ in $\mathfrak{g} / \mathfrak{h}$.

Notice that this is a major difference with the cotangent bundle case, where the cotangent-lifted action is free if and only if the base action is free as well. In the context of cosphere bundle reduction the reason for the special interest in semifree actions and in finding necessary and sufficient conditions for the freeness of the lifted cosphere action is the following. Given a cosphere bundle $\mathcal{C}=S^{*} Q$ with the lift of a proper almost semifree action on $Q$, if we ignore the bundle structure of the contact manifold $\mathcal{C}$ we are in the hypothesis of regular contact reduction, since $G$ acts freely, properly, and by strong contactomorphisms on $\mathcal{C}$. Therefore, the contact reduced space $\mathcal{C}_{0}$ is a well-defined smooth contact manifold.

On the other hand, since the action on $Q$ is not free in general, we cannot apply the main result on regular cosphere bundle reduction of [5] (see Theorem 3.1) because in that case the quotient $Q / G$ will not be a smooth manifold. In fact, one expects $\mathcal{C}_{0}$ to be a smooth reduced manifold fibrating continuously over the topological stratified space $Q / G$, but this bundle description cannot be achieved by only applying the scheme of regular cosphere bundle reduction. However, the results of the previous section will allow us to provide such a "stratified bundle" picture of the contact quotient $\mathcal{C}_{0}$. Indeed, we have the following result.

Theorem 6.1. Let $G$ be a Lie group acting properly and almost semifreely on $Q$ and by lifts on the cosphere bundle $S^{*} Q$ with contact momentum map $J: S^{*} Q \rightarrow \mathfrak{g}^{*}$. Write the orbit type decomposition of $Q / G$ as

$$
Q / G=Q^{(e)} \coprod_{(H) \in I_{Q} \backslash I_{Q}^{*}} *^{(H)},
$$

where $Q^{(e)}=Q_{(e)} / G$ is open and dense in $Q / G$ and each $*^{(H)}$ with $(H) \in I_{Q} \backslash I_{Q}^{*}$ is an isolated point of some lower-dimensional stratum $Q^{(H)}$ with $(H) \succ(e)$, lying in the boundary of $Q^{(e)}$. Then the quotient $\mathcal{C}_{0}=J^{-1}(0) / G$ is a smooth manifold which can be decomposed as

$$
\mathcal{C}_{0} \simeq S^{*} Q^{(e)} \coprod_{(H) \in I_{Q} \backslash I_{Q}^{*}} C S^{(H)},
$$

where each $C S^{(H)}$ is a trivial bundle over $*^{(H)}$ and a connected submanifold of $\mathcal{C}_{0}$ lying in the boundary of $S^{*} Q^{(e)}$. Moreover, the manifolds $C S^{(H)}$ are Legendrian submanifolds of $\mathcal{C}_{0}$ in one-to-one correspondence with the singular orbits of the $G$-action on $Q$ and have dimension $\operatorname{dim} Q-\operatorname{dim} G-1$. 
Proof. Since $J^{-1}(0)$ consists of a single orbit type (e), due to the fact that the lifted action to $S^{*} Q$ is free, the secondary and C-L stratifications coincide with the partition (6.1). As for every $(H) \in I_{Q}$ different from $(e)$ we have $(H) \in I_{Q} \backslash I_{Q}^{*}$, the contact seams $C S^{(H)}:=C S_{(H) \succ(e)}$ are Legendrian submanifolds of $\mathcal{C}_{0}$. The dimension of each connected component is then given by formula (5.5) noting that $\operatorname{dim} Q_{(e)}=\operatorname{dim} Q$ and $\operatorname{dim} Q^{(H)}=\operatorname{dim} Q_{(H)}-\operatorname{dim} G+\operatorname{dim} H=0$ for every $(H) \in$ $I_{Q} \backslash I_{Q}^{*}$, since the action on $Q$ is almost semifree.

Recall that a group action is called semifree if it is free everywhere except for a set of isolated fixed points. Semifree actions are important particular cases of almost semifree actions and they are commonly found in examples. The following example explicitly illustrates the geometric constructions of this paper in that situation.

Example: $S^{1}$ acting on $S^{*} \mathbb{R}^{2}$. Consider $Q=\mathbb{R}^{2}$ with Euclidean coordinates $\left(x_{1}, x_{2}\right)$ and its cotangent bundle $T^{*} \mathbb{R}^{2}=\mathbb{R}^{2} \times \mathbb{R}^{2}$ with coordinates $\left(x_{1}, x_{2}, y_{1}, y_{2}\right)$. The action of $S^{1}$ by rotations on $\mathbb{R}^{2}$ (a semifree action with $\mathbb{R}^{2}{ }_{\left(S^{1}\right)}=\{(0,0)\}$ ) lifts to $T^{*} \mathbb{R}^{2}$ by the induced diagonal action. A Hilbert basis for the ring of $S^{1}$-invariant polynomials for this cotangent lifted action is given by (see [3], §1.4)

$$
\begin{aligned}
\sigma_{1} & =x_{1}^{2}+x_{2}^{2}+y_{1}^{2}+y_{2}^{2}, \\
\sigma_{2} & =2\left(x_{1} y_{1}+x_{2} y_{2}\right), \\
\sigma_{3} & =y_{1}^{2}+y_{2}^{2}-x_{1}^{2}-x_{2}^{2}, \\
\sigma_{4} & =x_{1} y_{2}-x_{2} y_{1} .
\end{aligned}
$$

These polynomials satisfy the semialgebraic relations

$$
\sigma_{1} \geq 0, \quad \sigma_{1}^{2}=\sigma_{2}^{2}+\sigma_{3}^{2}+4 \sigma_{4}^{2} .
$$

We can identify the cosphere bundle $S^{*} \mathbb{R}^{2}$ with the subset of $T^{*} \mathbb{R}^{2}$ given by the constraint

$$
\sigma_{1}+\sigma_{3}=2 .
$$

The cotangent lifted action restricts to $S^{*} \mathbb{R}^{2}$ giving the free lifted action by contactomorphisms. Its associated momentum map is given by

$$
J\left(x_{1}, x_{2}, y_{1}, y_{2}\right)=\sigma_{4}
$$

for $\left(x_{1}, x_{2}, y_{1}, y_{2}\right) \in S^{*} \mathbb{R}^{2}$. Consequently, using invariant theory, the contact reduced space $J^{-1}(0) / S^{1}$ is identified with the semialgebraic variety of $\mathbb{R}^{3}=$ $\left\{\sigma_{2}, \sigma_{3}, \sigma_{1}\right\}$ defined by

$$
\mathcal{C}_{0} \simeq\left\{\left(\sigma_{2}, \sigma_{3}, \sigma_{1}\right) \in \mathbb{R}^{3}: \sigma_{1} \geq 0, \sigma_{1}^{2}=\sigma_{2}^{2}+\sigma_{3}^{2}, \sigma_{1}+\sigma_{3}=2\right\} .
$$

This contact reduced space is in fact a smooth manifold since it is the parabola obtained by intersecting the plane $P=\left\{\sigma_{1}+\sigma_{3}=2\right\}$ with the upper half of the cone $\sigma_{1}^{2}=\sigma_{2}^{2}+\sigma_{3}^{2}$ (see Figure 3). Its smooth structure is induced from the ambient space $\mathbb{R}^{3}$. This was to be expected since the action on the contact manifold $S^{*} \mathbb{R}^{2}$ is free.

However, this reduced space is no longer a cosphere bundle since the action on the base is semifree. We investigate now how the stratified bundle structure of $\mathcal{C}_{0}$ obtained in the previous sections arises here. Note that $Q / G=\mathbb{R}^{2} / S^{1}$ can be identified with the subset of $\mathbb{R}^{3}$ given by

$$
Q / G=\{(0,-t, t): t \geq 0\}
$$




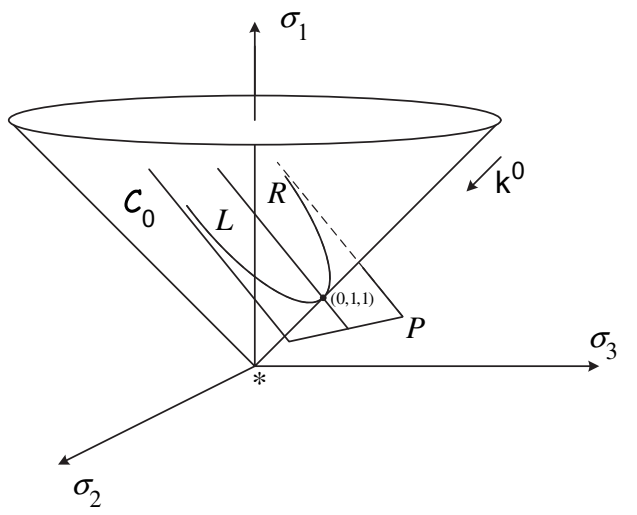

FiguRE 3. The contact reduced space as a parabola fibrating over a half-closed line

which is a half-open line parallel to the plane $P$ containing $\mathcal{C}_{0}$. According to the notation employed in this section, $Q / G$ is a stratified space with strata $Q^{(e)}$ and $*=(0,0,0)$. The continuous fibration $k^{0}: \mathcal{C}_{0} \rightarrow Q / G$ is given by $k^{0}\left(\sigma_{2}, \sigma_{3}, \sigma_{1}\right)=$ $\left(0,1-\sigma_{1}, \sigma_{1}-1\right)$. Note that $\left(k^{0}\right)^{-1}\left(Q^{(e)}\right)=L \coprod R$ and $\left(k^{0}\right)^{-1}(*)=(0,1,1)$ (see Figure 6), where $\mathcal{C}_{0}=L \coprod R \coprod\{(0,1,1)\}$. In addition, recall that $Q^{(e)} \simeq \mathbb{R}$ and that $S^{*} \mathbb{R}=\mathbb{R} \sqcup \mathbb{R}$.

So $\left(k^{0}\right)^{-1}\left(Q^{(e)}\right)=L \coprod R$ is diffeomorphic to the cosphere bundle $S^{*} Q^{(e)}$. The fiber over a point $(0,-t, t) \in Q^{(e)}$ is the pair of points $(2 \sqrt{t}, 1-t, 1+t)$ and $(-2 \sqrt{t}, 1-t, 1+t)$ which lie in $L$ and $R$ respectively. Finally, the point $(0,1,1)$, the minimum of the parabola $\mathcal{C}_{0}$, is the seam $C S_{\left(S^{1}\right) \succ(e)}$ lying in the boundary of $S^{*} Q^{(e)}$. Finally, since both $\mathcal{C}_{0}$ and $S^{*} Q^{(e)}$ are one-dimensional, their contact structures are trivial, due to the fact that the corresponding contact distributions must be zero-dimensional.

\section{Example: Diagonal toral aCtion on $\mathbb{R}^{2} \times \mathbb{R}^{2}$}

We illustrate the main results obtained in this paper with one more example rich enough to show all the extra structure appearing in the cosphere bundle singular reduction. This time, the reduced contact space $\mathcal{C}_{0}$ will have dimension greater than one and will have hence a non-trivial contact structure.

Consider the proper action of $G=\mathbb{T}^{2}$ on $Q=\mathbb{R}^{2} \times \mathbb{R}^{2}$, where each $S^{1}$ factor acts by rotations on the corresponding $\mathbb{R}^{2}$ factor. The isotropy lattice for this action is shown in Figure 4, where the subconjugation partial order is represented by arrows. Also, the corresponding stratification lattice is shown. A stratification lattice is a graphical arrangement of all the strata of a stratified space where for any two strata $A, B$ with $A \subseteq \bar{B}$ and such that there is no other stratum $C$ with the properties $A \subseteq \bar{C}$ and $C \subseteq \bar{B}$ we write $A \rightarrow B$. For the action under study, we have $I_{Q} \backslash I_{Q}^{*}=\left\{\left(\mathbb{T}^{2}\right)\right\}$.

Let $(x, y)=\left(x_{1}, x_{2}, y_{1}, y_{2}\right)$ be the Euclidean coordinates of a point in $Q$ and $z=$ $(x, y, u, v)=\left(x_{1}, x_{2}, y_{1}, y_{2}, u_{1}, u_{2}, v_{1}, v_{2}\right)$ the ones of a covector in $T^{*} Q \simeq \mathbb{R}^{4} \times \mathbb{R}^{4}$. 
The ring of $G$-invariant polynomials on $T^{*} Q$ is generated by

$$
\begin{array}{lll}
\rho_{1}=\|x\|^{2}+\|u\|^{2} & \sigma_{1}=\|y\|^{2}+\|v\|^{2} \\
\rho_{2}=2(x \cdot u) & \sigma_{2}=2(y \cdot v) \\
\rho_{3}=\|u\|^{2}-\|x\|^{2} & \sigma_{3}=\|v\|^{2}-\|y\|^{2} \\
\rho_{4}=x_{1} u_{2}-x_{2} u_{1} & \sigma_{4}=y_{1} v_{2}-y_{2} v_{1} .
\end{array}
$$

These polynomials, which form a Hilbert basis, are subject to the following semialgebraic relations:

$$
\rho_{1} \geq 0, \quad \sigma_{1} \geq 0, \quad \rho_{1}^{2}=\rho_{2}^{2}+\rho_{3}^{2}+4 \rho_{4}^{2}, \quad \sigma_{1}^{2}=\sigma_{2}^{2}+\sigma_{3}^{2}+4 \sigma_{4}^{2} .
$$

Identifying the cosphere bundle $S^{*} \mathbb{R}^{4}$ with $\mathbb{R}^{4} \times S^{3} \subset \mathbb{R}^{4} \times \mathbb{R}^{4}$, where $S^{3}=$ $\left\{(u, v) \in \mathbb{R}^{2} \times \mathbb{R}^{2}:\|u\|^{2}+\|v\|^{2}=1\right\}$, it is easy to see that its contact structure is given by the kernel of the restriction of the Liouville one-form $\theta=u d x+v d y$ and that the associated momentum map $J: S^{*} \mathbb{R}^{4} \rightarrow \mathbb{R}^{2}$ is given by $J(x, y, u, v)=$ $\left(\rho_{4}, \sigma_{4}\right) \in \mathbb{R}^{2}$. Consequently, we still have two more constraints to describe the zero-momentum level set:

$$
\rho_{4}=0 \quad \text { and } \quad \sigma_{4}=0 .
$$

Notice that we can also see $S^{*} \mathbb{R}^{4}$ as the subset of $\mathbb{R}^{8}$ defined by the additional constraint

$$
\rho_{1}+\rho_{3}+\sigma_{1}+\sigma_{3}=2 .
$$

The associated $G$-invariant Hilbert map is defined by

$$
\gamma: J^{-1}(0) \rightarrow \mathbb{R}^{3} \times \mathbb{R}^{3}, \quad \gamma(z)=\left(\rho_{1}(z), \rho_{2}(z), \rho_{3}(z) ; \sigma_{1}(z), \sigma_{2}(z), \sigma_{3}(z)\right),
$$

and we can identify the reduced contact space with the image of $\gamma$, i.e., with the semialgebraic variety of $\mathbb{R}^{6}$ defined by

$$
\mathcal{C}_{0} \simeq\left\{(\rho ; \sigma) \in \mathbb{R}^{6}: \rho_{1}, \sigma_{1} \geq 0, \rho_{1}^{2}=\rho_{2}^{2}+\rho_{3}^{2}, \sigma_{1}^{2}=\sigma_{2}^{2}+\sigma_{3}^{2}, \rho_{1}+\rho_{3}+\sigma_{1}+\sigma_{3}=2\right\}
$$

which is the intersection between the product of two cones, $C_{1} \times C_{2}$, and the hypersurface $H:=\left\{\left(\rho_{1}, \rho_{3}, \sigma_{1}, \sigma_{3}\right) \in \mathbb{R}^{4}: \rho_{1}+\rho_{3}+\sigma_{1}+\sigma_{3}=2\right\}$. (See Figure [5)

The Reeb vector field on $S^{*} \mathbb{R}^{4}$ is given by $R(x, y, u, v)=(u, v, 0,0)$ for any $(x, y, u, v) \in \mathbb{R}^{4} \times S^{3}$, and the flow of the corresponding reduced Reeb vector field on $\mathcal{C}_{0}$ at a point $\left(\rho_{\mathbf{0}} ; \sigma_{\mathbf{0}}\right)$ is easily computed as

$$
\begin{aligned}
& \rho_{1}(t)=\rho_{01}+\rho_{02} t+\frac{1}{2}\left(\rho_{01}+\rho_{03}\right) t^{2} \\
& \rho_{2}(t)=\rho_{02}+\left(\rho_{01}+\rho_{03}\right) t \\
& \rho_{3}(t)=\rho_{03}-\rho_{02} t-\frac{1}{2}\left(\rho_{01}+\rho_{03}\right) t^{2} \\
& \sigma_{1}(t)=\sigma_{01}+\sigma_{02} t+\frac{1}{2}\left(\sigma_{01}+\sigma_{03}\right) t^{2} \\
& \sigma_{2}(t)=\sigma_{02}+\left(\sigma_{01}+\sigma_{03}\right) t \\
& \sigma_{3}(t)=\sigma_{03}-\sigma_{02} t-\frac{1}{2}\left(\sigma_{01}+\sigma_{03}\right) t^{2} .
\end{aligned}
$$

Applying Proposition 4.1, we know that the orbit types of $J^{-1}(0)$ are exactly those given by $I_{Q}^{*}$ and hence the contact strata of $\mathcal{C}_{0}$ are in bijective correspondence 
with the strata of $Q$ given by $I_{Q}^{*}$. We then have

$$
\begin{array}{ll}
T^{*} Q_{\mathbf{e}} & =\left\{(x, y, u, v) \in \mathbb{R}^{8}:(x, y) \neq \mathbf{0}\right\} \\
T^{*} Q_{\left(S^{1} \times e\right)} & =\left\{(\mathbf{0}, y, \mathbf{0}, v) \in \mathbb{R}^{8}: y \neq \mathbf{0}\right\} \\
T^{*} Q_{e \times S^{1}} & =\left\{(x, \mathbf{0}, u, \mathbf{0}) \in \mathbb{R}^{8}: x \neq \mathbf{0}\right\} \\
N^{*} Q_{\mathbf{e}} & =\{(x, y, \mathbf{0}, \mathbf{0}): x \neq \mathbf{0}, y \neq \mathbf{0}\} \\
N^{*} Q_{S^{1} \times e} & =\{(\mathbf{0}, y, u, \mathbf{0}): y \neq \mathbf{0}\} \\
N^{*} Q_{e \times S^{1}} & =\{(x, \mathbf{0}, \mathbf{0}, v): x \neq \mathbf{0}\} .
\end{array}
$$

Consequently, a direct computation gives the following orbit types for the zero momentum map:

$$
\begin{aligned}
& J^{-1}(0) \quad=\left\{z \in \mathbb{R}^{4} \times S^{3}: \rho_{4}(z)=\sigma_{4}(z)=0\right\}, \\
& \left(J^{-1}(0)\right)_{(\mathbf{e})} \quad=\left\{z \in J^{-1}(0): x \neq 0, y \neq 0\right\} \\
& \coprod\left\{z \in J^{-1}(0): x=0, y \neq 0, u \neq 0\right\} \\
& \coprod\left\{z \in J^{-1}(0): x \neq 0, y=0, v \neq 0\right\} \\
& \coprod\left\{z \in\left\{0_{\mathbb{R}^{4}}\right\} \times S^{3}: u \neq 0, v \neq 0\right\}, \\
& \left(J^{-1}(0)\right)_{\left(e \times S^{1}\right)}=\left\{z \in J^{-1}(0): y=v=0, x \neq 0\right\} \\
& \coprod\left\{z \in J^{-1}(0): x=y=v=0,\|u\|=1\right\}, \\
& \left(J^{-1}(0)\right)_{\left(S^{1} \times e\right)}=\left\{z \in J^{-1}(0): x=u=0, y \neq 0\right\} \\
& \bigsqcup\left\{z \in J^{-1}(0): x=y=u=0,\|v\|=1\right\} .
\end{aligned}
$$

Using the image of the Hilbert map $\gamma$ we can realize the contact strata given by Theorems 2.1, 5.1, and 5.3 as:

$$
\begin{aligned}
& \mathcal{C}_{0}^{(\mathbf{e})} \quad=C C_{(\mathbf{e})} \amalg C S_{\left(S^{1} \times e\right) \succ(\mathbf{e})} \amalg C S_{\left(e \times S^{1}\right) \succ(\mathbf{e})} \amalg C S_{\left(\mathbb{T}^{2}\right) \succ(\mathbf{e})}, \\
& C C_{(\mathrm{e})} \quad=\left\{(\rho ; \sigma): \rho_{1}, \sigma_{1}>0, \rho_{1} \neq \rho_{3}, \sigma_{1} \neq \sigma_{3}, \rho_{1}^{2}=\rho_{2}^{2}+\rho_{3}^{2},\right. \\
& \left.\sigma_{1}^{2}=\sigma_{2}^{2}+\sigma_{3}^{2}, \rho_{1}+\rho_{3}+\sigma_{1}+\sigma_{3}=2\right\} \text {, } \\
& C S_{\left(S^{1} \times e\right) \succ(\mathbf{e})}=\left\{(\rho ; \sigma): \rho_{1}, \sigma_{1}>0, \sigma_{1} \neq \sigma_{3}, \rho_{1}=\rho_{3}, \rho_{2}=0,\right. \\
& \left.2 \rho_{1}+\sigma_{1}+\sigma_{3}=2, \sigma_{1}^{2}=\sigma_{2}^{2}+\sigma_{3}^{2}\right\} \\
& =\left(\mathbb{R}_{+} \times C_{2}\right) \cap\left\{2 \rho_{1}+\sigma_{1}+\sigma_{3}=2, \sigma_{1} \neq \sigma_{3}\right\}, \\
& C S_{\left(e \times S^{1}\right) \succ(\mathbf{e})}=\left\{(\rho ; \sigma): \rho_{1}, \sigma_{1}>0, \rho_{1} \neq \rho_{3}, \sigma_{1}=\sigma_{3}, \sigma_{2}=0,\right. \\
& \left.2 \sigma_{1}+\rho_{1}+\rho_{3}=2, \rho_{1}^{2}=\rho_{2}^{2}+\rho_{3}^{2}\right\} \\
& =\left(C_{1} \times \mathbb{R}_{+}\right) \cap\left\{2 \sigma_{1}+\rho_{1}+\rho_{3}=2, \rho_{1} \neq \rho_{3}\right\} \text {, } \\
& C S_{\left(\mathbb{T}^{2}\right) \succ(\mathbf{e})} \quad=\left\{(\rho ; \sigma): \rho_{1}, \sigma_{1}>0, \rho_{1}=\rho_{3}, \sigma_{1}=\sigma_{3}, \rho_{2}=\sigma_{2}=0,\right. \\
& \left.\rho_{1}+\sigma_{1}=1\right\}, \\
& \mathcal{C}_{0}^{\left(e \times S^{1}\right)} \quad=C C_{\left(e \times S^{1}\right)} \amalg C S_{\left(\mathbb{T}^{2}\right) \succ\left(e \times S^{1}\right)}, \\
& C C_{\left(e \times S^{1}\right)}=\left\{(\rho ; \mathbf{0}): \rho_{1}>0, \rho_{1}+\rho_{3}=2, \rho_{1}^{2}=\rho_{2}^{2}+\rho_{3}^{2}\right\} \backslash\{(1,0,1 ; \mathbf{0})\}, \\
& C S_{\left(\mathbb{T}^{2}\right) \succ\left(e \times S^{1}\right)}=\{(1,0,1 ; 0,0,0)\}, \\
& \mathcal{C}_{0}^{\left(S^{1} \times e\right)} \quad=C C_{\left(S^{1} \times e\right)} \amalg C S_{\left(\mathbb{T}^{2}\right) \succ\left(S^{1} \times e\right)}, \\
& C C_{\left(S^{1} \times e\right)}=\left\{(\mathbf{0} ; \sigma): \sigma_{1}>0, \sigma_{1}+\sigma_{3}=2, \sigma_{1}^{2}=\sigma_{2}^{2}+\sigma_{3}^{2}\right\} \backslash\{(\mathbf{0} ; 1,0,1)\}, \\
& C S_{\left(\mathbb{T}^{2}\right) \succ\left(S^{1} \times e\right)}=\{(0,0,0 ; 1,0,1)\} .
\end{aligned}
$$

The corresponding contact, secondary and C-L stratification lattices in $\mathcal{C}_{0}$ are shown in Figure 6. Notice that (e) is the principal orbit type in $Q$ and, therefore, $C C_{(\mathbf{e})}$ is open and dense in the reduced space $\mathcal{C}_{0}$. The contact seams $C S_{\left(\mathbb{T}^{2}\right) \succ\left(S^{1} \times e\right)}$, $C S_{\left(\mathbb{T}^{2}\right) \succ\left(e \times S^{1}\right)}$, and $C S_{\left(\mathbb{T}^{2}\right) \succ(\mathbf{e})}$ are Legendrian submanifolds of their contact strata, 

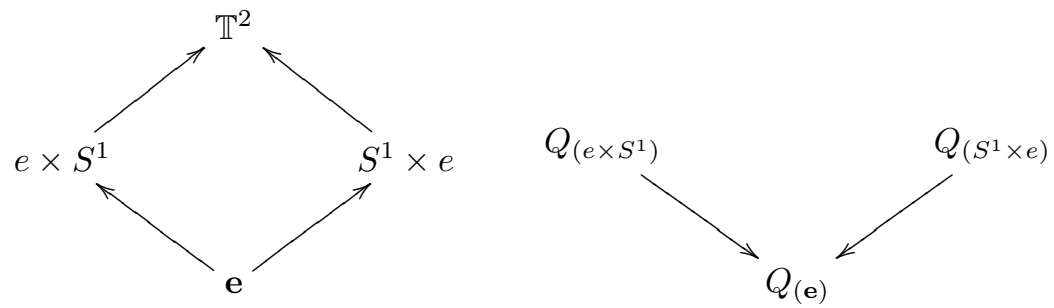

FIgURE 4. Isotropy and stratification lattices for the $\mathbb{T}^{2}$ action on $\mathbb{R}^{4}$
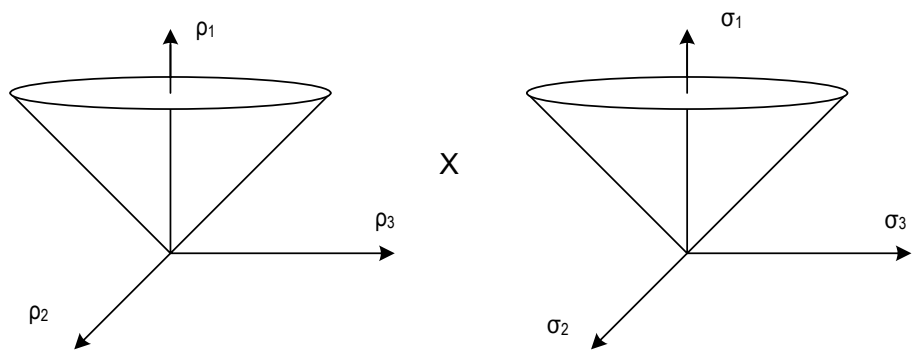

FiguRE 5. The ambient space of $\mathcal{C}_{0}$

while the rest are coisotropic. Every contact seam is mapped by the flow of the reduced Reeb vector field into the CC-secondary stratum of its corresponding contact stratum as can be easily checked.

In order to understand the bundle structure of these stratifications, we embed $Q$ in $T^{*} Q$ as the zero section and we identify $Q / G$ with the subset of the image of $\gamma$ given by

$$
Q / G=\left\{\left(t_{1}, 0,-t_{1} ; t_{2}, 0,-t_{2}\right): t_{1}, t_{2} \geq 0\right\} \simeq \mathbb{R}_{+} \times \mathbb{R}_{+},
$$

a half-plane parallel to $H$. The strata of its orbit stratification are

$$
\begin{aligned}
& Q^{\left(e \times S^{1}\right)}=\left\{\left(t_{1}, 0,-t_{1} ; \mathbf{0}\right): t_{1}>0\right\} \\
& Q^{\left(S^{1} \times e\right)}=\left\{\left(\mathbf{0} ; t_{2}, 0,-t_{2}\right): t_{2}>0\right\} \\
& Q^{(\mathbf{e})}=\left\{\left(t_{1}, 0,-t_{1} ; t_{2}, ; 0,-t_{2}\right): t_{1}, t_{2}>0\right\}
\end{aligned}
$$

and we obtain that the corresponding cosphere-like strata of $\mathcal{C}_{0}$ are diffeomorphic to the cosphere bundles

$$
S^{*} Q^{\left(e \times S^{1}\right)} \simeq S^{*} Q^{\left(S^{1} \times e\right)} \simeq \mathbb{R} \sqcup \mathbb{R} \quad \text { and } \quad S^{*} Q^{(\mathbf{e})} \simeq \mathbb{R}^{2} \times S^{1} .
$$

The continuous fibration $k^{0}: \mathcal{C}_{0} \rightarrow Q / G$ is given by $k^{0}\left(\rho_{1}, \rho_{2}, \rho_{3} ; \sigma_{1}, \sigma_{2}, \sigma_{3}\right)=$ $\left(\rho_{1}-1,0,1-\rho_{1} ; \sigma_{1}-1,0,1-\sigma_{1}\right)$. 


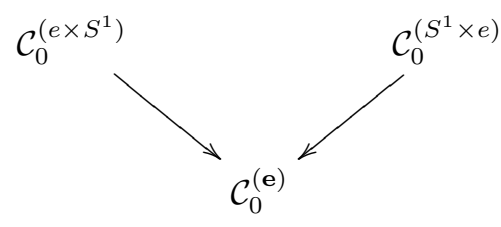

a)

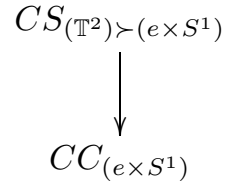

b)

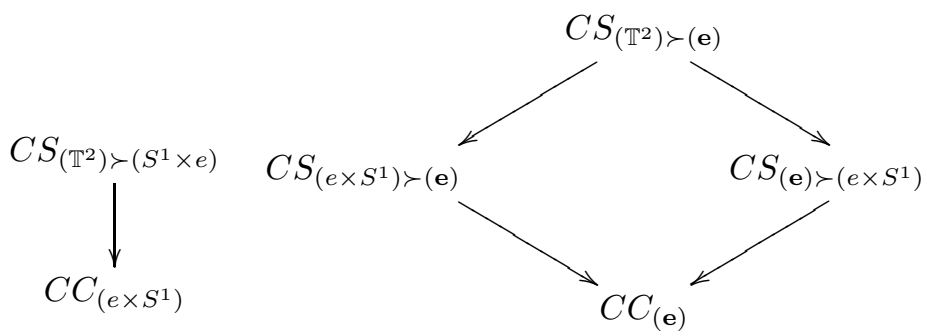

c)

d)

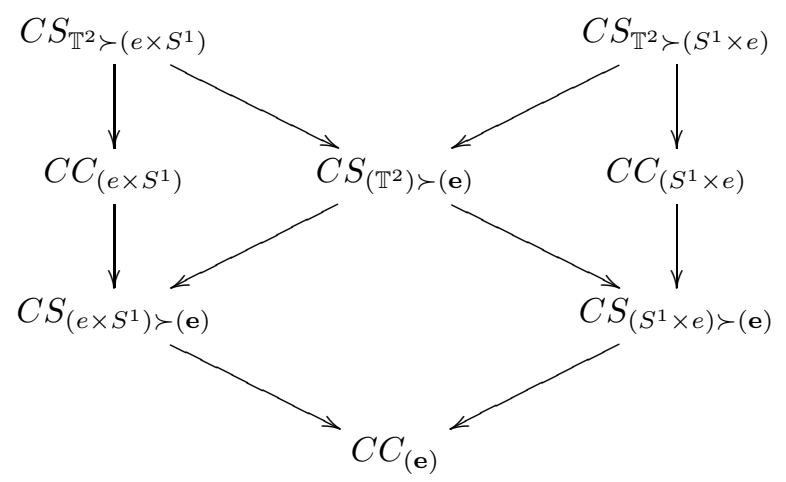

e)

FiguRE 6. a) Contact stratification of $\mathcal{C}_{0}$. Secondary stratifications of: b) $\mathcal{C}_{0}^{\left(e \times S^{1}\right)}$, c) $\mathcal{C}_{0}^{\left(S^{1} \times e\right)}$ and d) $\mathcal{C}_{0}^{(\mathbf{e})}$. e) CoisotropicLegendrian stratification of $\mathcal{C}_{0}$.

\section{REFERENCES}

[1] C. Albert, Le théorème de réduction de Marsden-Weinstein en géométrie cosymplectique et de contact, J. Geom. Physics, 6 (1989), 627-649. MR1076705 (91k:58033)

[2] D.E. Blair, Riemannian geometry of contact and symplectic manifolds, Progress in Math. 203, Birkhäuser, Boston, Basel, 2002. MR1874240 (2002m:53120)

[3] R.H. Cushman and L.M. Bates, Global Aspects of Classical Integrable Systems, Birkhäuser, Basel, 1997. MR1438060 (98a:58083)

[4] O. Drăgulete, L. Ornea, Non-zero Contact and Sasakian Reduction, Differential Geom. Appl. 24 (2006), 260-270. MR2216940 
[5] O. Drăgulete, L. Ornea, T.S. Ratiu, Cosphere bundle reduction in contact geometry, J. Symplectic Geom., 1 (2003), 695-714. MR2039161 (2004m:53141)

[6] J. J. Duistermaat, J. A. Kolk, Lie Groups, Universitext, Springer-Verlag, 2000. MR1738431 (2001j:22008)

[7] T. Ekholm, J. B. Etnyre, Invariants of Knots, Embeddings and Immersions via Contact Geometry, math.GT/0412517. MR2189927

[8] H. Geiges, Constructions of contact manifolds, Math. Proc. Cambridge Philos. Soc., 121 (1997), 455-464. MR1434654 (98f:53027)

[9] H. Geiges, A Brief History of Contact Geometry and Topology, Expo. Math., 19 (2001), 25-53. MR.1820126(2002c:53129)

[10] V. Guillemin and S. Sternberg, Homogeneous quantization and multiplicities of group representations, J. Funct. Anal., 47 (1982), 344-380. MR0665022 (84d:58034)

[11] E. Lerman, Contact cuts, Israel J. Math., 124 (2001), 77-92. MR.1856505 (2002g:53156)

[12] E. Lerman, C. Willett, The topological structure of contact and symplectic quotients, Internat. Math. Res. Notices, 1 (2001), 33-52. MR1809496 (2001j:53112)

[13] F. Loose, Reduction in contact geometry, J. Lie Theory, 11, no 1. (2001), 9-22. MR1828281 (2002g:53147)

[14] J.E. Marsden, T.S. Ratiu, Introduction to Mechanics and Symmetry, Springer Texts in Appl. Math. 17, Second edition, Second printing 2003. MR1304682 (95i:58073)

[15] J-P. Ortega and T.R. Ratiu, Momentum Maps and Hamiltonian Reduction, Progress in Mathematics, Volume 222, Birkhäuser, Boston, 2004. MR2021152 (2005a:53144)

[16] R.S. Palais, On the existence of slices for actions of non-compact Lie groups, Ann. of Math. (2), 73 (1961), 295-323. MR0126506 (23:A3802)

[17] M. Perlmutter, M. Rodríguez-Olmos, M. E. Sousa-Dias, On the geometry of reduced cotangent bundles at zero momentum, math.SG/0310437.

[18] M. J. Pflaum, Analytic and Geometric Study of Stratified Spaces, Lecture Notes in Mathematics, volume 510, Springer Verlag, 2001. MR1869601 (2002m:58007)

[19] T. Ratiu, R. Schmid, The differentiable structure of three remarkable diffeomorphism groups, Math. Z., 177 (1981), 81-100. MR0611471 (83d:57024)

[20] C. Willett, Contact reduction, Trans. Amer. Math. Soc., 354 (2002), 4245-4260. MR.1926873 (2003m:53152)

[21] M. Zambon, C. Zhu, Contact reduction and groupoid actions, math.DG/0405047.

Section de mathématiques, EPFL, CH-1015 Lausanne, Switzerland and Department of Mathematics, University "Politehnica" of Bucharest, Romania

E-mail address: oana.dragulete@epfl.ch

Section de mathématiques, EPFL, CH-1015 Lausanne, Switzerland

E-mail address: tudor.ratiu@epfl.ch

Section de mathématiques, EPFL, CH-1015 Lausanne, Switzerland

E-mail address: miguel.rodriguez@epfl.ch 